\title{
A Secure and Efficient Authenticated Diffie-Hellman Protocol
}

\author{
Augustin P. Sarr ${ }^{1}$, Philippe Elbaz-Vincent ${ }^{2}$, and Jean-Claude Bajard ${ }^{3}$ \\ ${ }^{1}$ Netheos R\&D \\ ${ }^{1,2}$ Institut Fourier - CNRS, Université Grenoble 1 \\ ${ }^{3}$ LIP6 - CNRS, Université Pierre et Marie Curie
}

\begin{abstract}
The Exponential Challenge Response (XRC) and Dual Exponential Challenge Response (DCR) signature schemes are the building blocks of the HMQV protocol. We propose a complementary analysis of these schemes; on the basis of this analysis we show how impersonation and man in the middle attacks can be mounted against the HMQV protocol when some session specific information leakages happen. We define the Full Exponential Challenge Response (FXRC) and Full Dual Exponential Challenge Response (FDCR) signature schemes; using these schemes we propose the Fully Hashed MQV protocol (with security arguments), which preserves the remarkable performance of the (H)MQV protocols and resists the attacks we present.
\end{abstract}

\section{Introduction}

Implicitly authenticated key exchange protocols have gained wide acceptance; in addition to providing implicit authentication, these protocols are usually more efficient than the explicitly authenticated ones. The HMQV protocol [11], inspired by the famous MQV protocol [14, 1, 2, $9,10,20,8]$, was proposed with security arguments in the Canetti-Krawczyk model [5]. HMQV was designed in accord with the principle that "a good security system is not one that denies the possibility of failures but rather one designed to confine the adverse effects of such failures to the possible minimum" [11]. Session secret leakages may happen; in that case the exposed session may be compromised, but this should have no effect on the security of any other unexposed session.

In this paper, we propose a complementary analysis of the Exponential Challenge Response (XCR) and Dual Exponential Challenge Response (DCR) signature schemes. On the basis of this analysis we show how impersonation and man in the middle attacks can be performed against HMQV when some session specific information leakages happen. We propose the Full Exponential Challenge Response (FXRC) and Full Dual Exponential Challenge Response (FDCR) signature schemes. With these schemes we define the Fully Hashed MQV protocol (with security arguments), which resists the attacks we present and preserves the remarkable performance of the (H)MQV protocol.

This paper is organized as follows. In section 2 we analyze some aspects of the XCR and DCR signatures schemes; we show how session specific information leakages can be used for impersonation and man in the middle attacks against $(\mathrm{H}) \mathrm{MQV}$. In section 3 we define a CanettiKrawczyk type security model $[5,13]$ for the $(\mathrm{H}) \mathrm{MQV}$ type protocols. In section 4, we propose the FXCR and FDCR signature schemes; and using these schemes we propose the FHMQV protocol. Section 5 deals with the FHMQV security arguments; in section 6 we present the FHMQV-C protocol (the 'C' stands for key confirmation), which provides additional security attributes, namely key confirmation and perfect forward secrecy. We conclude in section 7 .

The following notations are used in this paper: $\mathcal{G}$ is a multiplicatively written cyclic group of prime order $q$ generated by $G,|q|$ is the bit length of $q$. The identity element in $\mathcal{G}$ is denoted $\overline{1}$, 
and $\mathcal{G}^{*}$ is the set of non-identity elements in $\mathcal{G}$; all public keys are supposed to belong to $\mathcal{G}^{*}$. For a group element $X \in \mathcal{G}$, the lowercase $x$ denotes the discrete logarithm of $X$ in base $G$. The identity of an entity with public key $A$ (and private key $a$ ) is denoted $\hat{A}$ ( $\hat{A}$ is supposed to contain $A$, or sufficient information to learn $A$ ); For two identities $\hat{A} \neq \hat{B}$, we suppose that no substring of $\hat{A}$ equals $\hat{B}$, and conversely. $H$ is a $\lambda$-bit hash function where $\lambda$ is the length of the desired session key, and $\bar{H}$ is a $l$-bit hash function where $l=\left(\left\lfloor\log _{2} q\right\rfloor+1\right) / 2$ (see [11, Remark 4.2] for a discussion on the value of $l$ ); the concatenation of $n$ strings $s_{1}, \ldots, s_{n}$ is denoted $\left(s_{1}, \ldots, s_{n}\right)$. The symbol " $\in_{R}$ " stands for "chosen uniformly at random in." The Computational Diffie-Hellman $(\mathrm{CDH})$ assumption is supposed to hold in $\mathcal{G}$, i.e. given $U=G^{u}$ and $V=G^{v}$ with $U, V \in_{R} \mathcal{G}^{*}$, computing $C D H(U, V)=G^{u v}$ is infeasible.

\section{Complementary Analysis of the HMQV design}

We show in this section how session specific information leakages, can be used for impersonation and man in the middle attacks against HMQV.

Note. In our description of HMQV, the ephemeral public keys are tested for membership in $\mathcal{G}^{*}$. Ephemeral public key validation is voluntarily omitted in [11], but the HMQV protocol is known to be insecure if ephemeral keys are not correctly validated $[18,17]$.

\subsection{Exploiting Secret Leakage in the XCR and DCR Signature Schemes}

Definition 1 (Exponential Challenge-Response Signature [11]). Let $\hat{B}$ be an entity with public key $B \in \mathcal{G}^{*}$, and $\hat{A}$ a verifier. $\hat{B}$ 's signature on a message $m$ and challenge $X$ provided by $\hat{A}$ $\left(X=G^{x}, x \in_{R}[1, q-1]\right.$ is chosen and kept secret by $\left.\hat{A}\right)$ is $\operatorname{Sig}_{\hat{B}}(m, X)=\left(Y, X^{s_{B}}\right)$, where $Y=$ $G^{y}, y \in_{R}[1, q-1]$ is chosen by $\hat{B}$, and $s_{B}=y+\bar{H}(Y, m) b$. The verifier $\hat{A}$ accepts a pair $\left(Y, \sigma_{B}\right)$ provided by $\hat{B}$ as a valid signature if $Y \in \mathcal{G}^{*}$ and $\left(Y B^{e}\right)^{x}=\sigma_{B}$, where $e=\bar{H}(Y, m)$.

In this scheme, the information $s_{B}$ "allows" an attacker to generate valid signatures. Indeed, given the $s_{B}$, "corresponding" to some message $m$ and some challenge $Y$, one can generate a valid signature on any message-challenge pair $\left(m, X_{1}\right)\left(X_{1}\right.$ is a new challenge and the message is unchanged). In a $(\mathrm{H}) \mathrm{MQV}^{1}$ session between $\hat{A}$ and $\hat{B}$, the identity of $\hat{B}$ stands for $\hat{A}$ 's message to $\hat{B}$, and thus does not change from one session (between $\hat{A}$ and $\hat{B}$ ) to another; this can be exploited when $s_{B}$ leakage happens.

Proposition 1. Let $\hat{B}$ be an entity, with public key $B \in \mathcal{G}^{*}$, signing a message-challenge pair $(m, X)$. If an attacker learns the $\beta$ most significant bits of $s_{B}$, then it can generate a valid signature with respect to $\hat{B}$ 's public key, on any message-challenge pair $\left(m, X_{1}\right)$ (the message is unchanged); this requires $\mathcal{O}\left(2^{\frac{|q|-\beta}{2}}\right)$ time complexity and $\mathcal{O}\left(2^{\frac{|q|-\beta}{2}}\right)$ space complexity.

Proof. From Shank's Baby Step Giant Step (BSGS) lemma [25], given $\sigma_{B}=X^{s_{B}}, X \in\langle G\rangle$, and the $\beta$ most significant bits of $s_{B}$, one can compute $s_{B}$ in $\mathcal{O}\left(2^{\frac{|q|-\beta}{2}}\right)$ time complexity and $\mathcal{O}\left(2^{\frac{|q|-\beta}{2}}\right)$ space complexity. Hence given a message-challenge pair $\left(m, X_{1}\right)$, the attacker replays $Y$ (since $Y$ is chosen by the signer) and produces $\left(Y, X_{1}^{s_{B}}\right)$ as a signature.

Shank's method is deterministic, but requires a large storage; using the Pollard's Kangaroo method $[24,25]$ one can compute $s_{B}$ with negligible storage in probabilistic run time $\mathcal{O}\left(2^{\frac{|q|-\beta}{2}}\right)$.

Definition 2 (Dual XCR Signature [11]). Let $\hat{A}$ and $\hat{B}$ be two parties with keys $A, B \in$ $\mathcal{G}$; and $m_{1}, m_{2}$ two messages. The Dual XCR (DCR) signature of $\hat{A}$ and $\hat{B}$ on $m_{1}, m_{2}$ is $\operatorname{DSig}_{\hat{A}, \hat{B}}\left(m_{1}, m_{2}, X, Y\right)=G^{(x+d a)(y+e b)}$, where $X=G^{x}, Y=G^{y} \in_{R} \mathcal{G}^{*}$ are chosen respectively by $\hat{A}$ and $\hat{B}, d=\bar{H}\left(X, m_{1}\right)$, and $e=\bar{H}\left(Y, m_{2}\right)$.

\footnotetext{
${ }^{1}$ When regarded through a XCR scheme, the XCR variant corresponding to MQV does not use a message in the computation of $s_{B}\left(s_{B}=y+\bar{Y} b\right.$, with $\bar{Y}=2^{l}+\left(\dot{Y} \bmod 2^{l}\right)$ where $\dot{Y}$ is the integer representation of $\left.Y\right)$, and thus can be analyzed as if it takes a constant message.
} 
The DCR signature of $\hat{A}$ and $\hat{B}$ on messages $m_{1}, m_{2}$ is a XCR signature of $\hat{A}$ on $m_{1}$ and challenge $Y B^{e}$. Hence, an attacker which learns the $\beta$ most significant bits of $s_{A}=x+d a$ can, for any message $m_{2}^{\prime}$, and any challenge $Y^{\prime}$ from $\hat{B}$, compute a valid DCR signature of $\hat{A}$ and $\hat{B}$ on messages $m_{1}, m_{2}^{\prime}$ and challenges $X, Y^{\prime}$. This requires $\mathcal{O}\left(2^{\frac{|q|-\beta}{2}}\right)$ time complexity and $\mathcal{O}\left(2^{\frac{|q|-\beta}{2}}\right)$ space complexity.

\subsection{Exploiting Session Secret Leakages in the HMQV Protocol}

A HMQV key exchange between two parties $\hat{A}$ and $\hat{B}$ is as in Protocol 1; if any verification fails, the execution aborts.

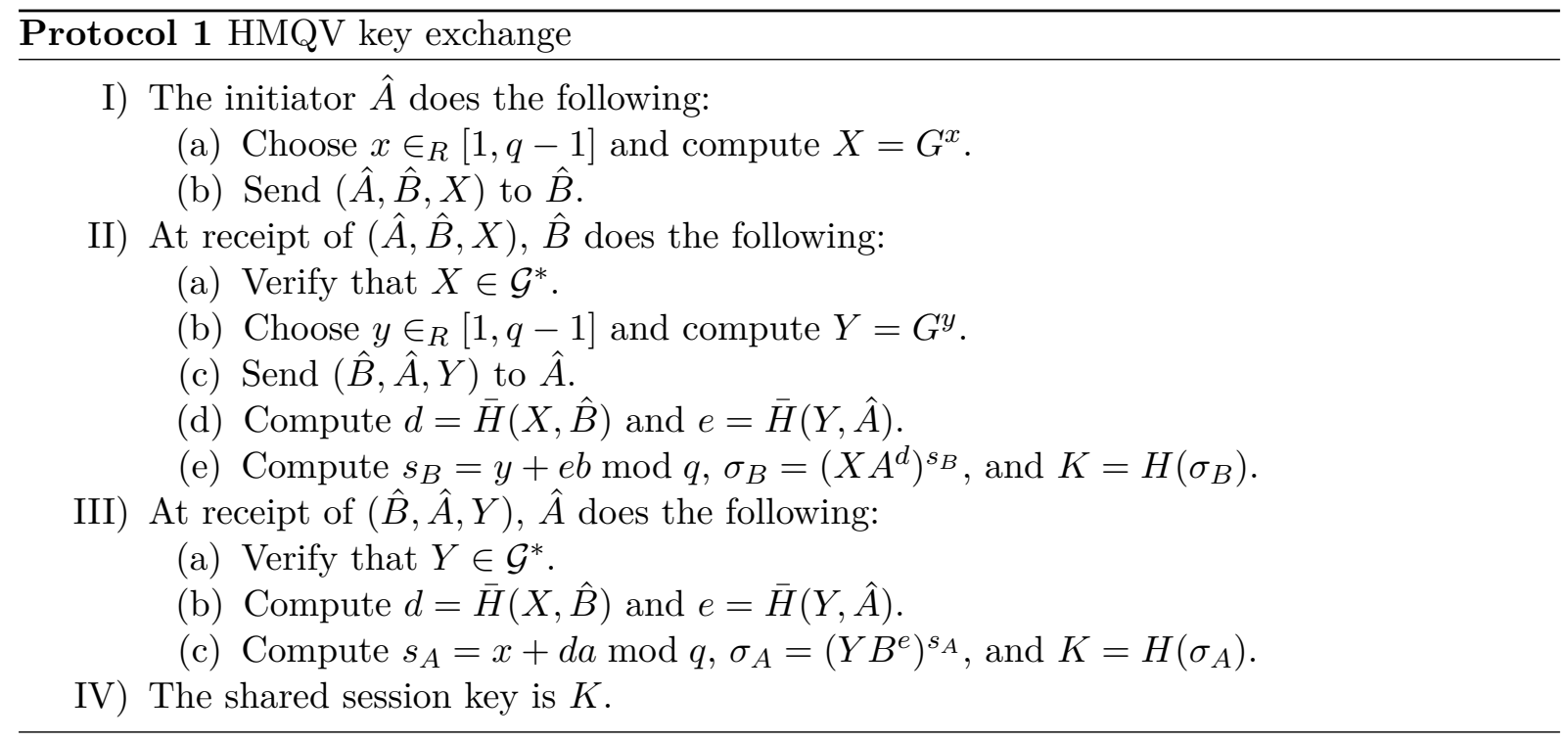

Roughly speaking, the secret shared between $\hat{A}$ and $\hat{B}$ is a DCR signature with messages fixed to $\hat{A}$ and $\hat{B}$. In [11], Krawczyk presents the XCR scheme as a new variant of the following Schnorr's identification scheme:

(a) The signer $\hat{B}$ chooses $y \in_{R}[1, q-1]$ and sends $Y=G^{y}$ to $\hat{A}$.

(b) The verifier $\hat{A}$ chooses $e \in_{R}[1, q-1]$ and sends $e$ to $\hat{B}$.

(c) $\hat{B}$ computes $s=y+e b$ and sends $s$ to $\hat{A}$.

(d) $\hat{A}$ accepts $s$ as a valid signature if $Y \in \mathcal{G}^{*}$ and $G^{s}=Y B^{e}$.

There is however a subtlety: in Schnorr's scheme the random element $e$, used by $\hat{B}$ when computing $s$, is always provided by the verifier $\hat{A}$; while in the XCR and DCR schemes, when $\hat{A}$ 's message $m_{1}$ is fixed (to $\hat{B}$ as in all the sessions between $\hat{A}$ and $\hat{B}$ ) the value of $e$, used when computing $s_{B}$, depends only on the ephemeral key $Y$ provided by (the signer) $\hat{B}$. This is what makes the replay attacks possible against the XCR and DCR schemes, and the $(\mathrm{C}, \mathrm{H}) \mathrm{MQV}(-\mathrm{C})$ protocols, when ephemeral secret exponent leakage happens.

\subsubsection{Impersonation Attack using Session Secret Leakage}

We show in this section how ephemeral secret exponent $\left(s_{A}\right.$ or $\left.s_{B}\right)$ leakage can be used for impersonation attack ${ }^{2}$. The following definition gives a broader view of the points needed for impersonation attack; these points are recalled to make the analysis reading easier.

\footnotetext{
${ }^{2}$ This attack is also reported in [3] (Appendix C). This work is however independent from [3], as we submitted at WCC 2009 (on February 9th, 2009) a paper (\#1569187679) which describe this attack, therefore before [3] was posted at http://eprint.iacr.org/2009/079 (on February 12th, 2009).
} 
Definition 3 (Point for impersonation attack, $i$-point). Let $\hat{A}$ and $\hat{B}$ be two entities with respective keys $A, B \in \mathcal{G}^{*}$. A group element $R \in \mathcal{G}^{*}$ is said to be a HMQV $i$-point for $\hat{A}$ to $\hat{B}$ if there exists some $k \in[1, q-1]$ such that $R=G^{k} A^{-\bar{H}(R, \hat{B})} ; k$ is said to be the decomposition.

Proposition 2. Let $\mathcal{G}=\langle G\rangle$ be a group with prime order $q, \hat{A}$ and $\hat{B}$ two entities with respective keys $A, B \in \mathcal{G}^{*}$. There is at least $q-\left(2^{l}+1\right) H M Q V i$-points for $\hat{A}$ to $\hat{B}$.

Proof. Let $\overline{\mathcal{G}}$ be the image of $\mathcal{G} \operatorname{through}(R \stackrel{\bar{H}}{\longrightarrow} \bar{R}=\bar{H}(R, \hat{B}))$. Since $\bar{H}$ is a $l$-bit hash function, the cardinal of $\overline{\mathcal{G}}$ is $\leqslant 2^{l}$. For every $\bar{Y} \in \overline{\mathcal{G}}$ there is at most one element $R_{0} \in \mathcal{G}$ such that $\bar{R}_{0}=\bar{Y}$ and $R_{0} A^{\bar{R}_{0}}=\overline{1}$; since the existence of another element $R_{0}^{\prime} \in \mathcal{G}$, which satisfies $\bar{R}_{0}^{\prime}=\bar{Y}$ and $R_{0}^{\prime} A^{\bar{R}_{0}^{\prime}}=\overline{1}$, would imply $R_{0} A^{\bar{Y}}=R_{0}^{\prime} A^{\bar{Y}}$ i.e. $R_{0}^{\prime}=R_{0}$.

Let $\mathcal{R}_{0}$ be the set of such $R_{0}$ points. The cardinal of $\mathcal{R}_{0}$ is at most $2^{l}$. Every element $R \in \mathcal{G}^{*} \backslash \mathcal{R}_{0}$ is a HMQV $i$-point for $\hat{A}$ to $\hat{B}$. Indeed for a such element $R, R A^{\bar{R}} \neq \overline{1}$ and since $R$ and $A$ are in $\mathcal{G}^{*}$, there exists some $k \in[1, q-1]$ such that $R A^{\bar{R}}=G^{k}$, or equivalently $R=G^{k} A^{-\bar{R}}$. Hence there is at least $q-\left(2^{l}+1\right)$ HMQV $i$-points for $\hat{A}$ to $\hat{B}$.

As shown in Attack 2 given a HMQV $i$-point for $\hat{A}$ to $\hat{B} X^{\prime}$ and its decomposition $k$, one can impersonate $\hat{A}$ to $\hat{B}$ with no more computations than needed by a HMQV execution. Notice that the important aspect is knowing the decomposition of an $i$-point.

Attack 2 HMQV impersonation of $\hat{A}$ to $\hat{B}$

Require: A HMQV $i$-point for $\hat{A}$ to $\hat{B} X^{\prime}$ and its decomposition $k$.

(1) Send $\left(\hat{A}, \hat{B}, X^{\prime}\right)$ to $\hat{B}$.

(2) Intercept $\hat{B}$ 's message to $\hat{A}(\hat{B}, \hat{A}, Y)$ and do the following:

(a) Verify that $Y \in \mathcal{G}^{*}$.

(b) Compute $\sigma_{A}=\left(Y B^{e}\right)^{k}$ where $e=\bar{H}(Y, \hat{A})$.

(c) Compute $K=H\left(\sigma_{A}\right)$.

(3) Use $K$ to communicate with $\hat{B}$ on behalf of $\hat{A}$.

A naive approach for decomposed $i$-point for $\hat{A}$ to $\hat{B}$ search consists in choosing $u \in\{0,1\}^{l}$ and computing the $2^{l}$ points $R_{k u}=G^{k} A^{-u}$, for $k=1, \ldots, 2^{l}$. If the hash function $\bar{H}$ is supposed to be a random oracle, the probability that $\bar{H}\left(R_{k u}, \hat{B}\right)$ equals $u$ is $\operatorname{Pr}\left(\bar{H}\left(R_{k u}, \hat{B}\right)=u\right)=1 / 2^{l}$. The number of successes $\left(R_{k u}: \bar{H}\left(R_{k u}, \hat{B}\right)=u\right)$ in these computations is a binomial random variable with parameters $\left(2^{l}, 1 / 2^{l}\right)$; hence these computations lead to a decomposed $i$-point with probability $\operatorname{Pr} s=1-\left(1-1 / 2^{l}\right)^{2^{l}} \approx 1-e^{-1} \approx 0.63>1 / 2$ for $l$ sufficiently large. Pollard's rho algorithm $[24,26,25]$ can be modified to take into account decomposed $i$-points detection, the resulting algorithm produces either a decomposed $i$-point or a discrete logarithm; this approach is expected to duplicate the efficiency of the rho algorithm.

It is worthwhile to mention that the MQV variant wherein the shared secret between two parties $\hat{A}$ and $\hat{B}$ is computed as $\sigma=\left(X A^{\bar{H}(X)}\right)^{y+\bar{H}(Y) b}=\left(Y B^{\bar{H}(Y)}\right)^{x+\bar{H}(X) a}$ (and the session as $K=H(\sigma))$ presents the following unfortunate aspect. If an attacker finds $x_{0} \in[1, q-1]$ such that $H\left(G^{x_{0}}\right)=0$, then it can impersonate any entity to any other entity. Finding such an $x_{0}$ requires $\mathcal{O}\left(2^{l}\right)$ operations. To impersonate a party, say $\hat{A}$, to a party $\hat{B}$ the attacker sends $\left(\hat{A}, \hat{B}, X_{0}\right)$ to $\hat{B}$, intercepts $\hat{B}$ 's message to $\hat{B}$, and computes $\sigma=\left(Y B^{\bar{H}(Y)}\right)^{x_{0}}$ and $K=H(\sigma)$; it then uses $K$ to communicate with $\hat{B}$ on behalf of $\hat{A}$.

In the following proposition, we link partial ephemeral secret exponent leakage to impersonation attack.

Proposition 3. Let $\hat{A}$ be an entity executing the $H M Q V$ protocol with some peer $\hat{B}$. If an attacker learns the $\beta$ most significant bits of the ephemeral secret exponent at $\hat{A}$, then it can 
indefinitely impersonate $\hat{A}$ to $\hat{B}$. This requires $\mathcal{O}\left(2^{\frac{|q|-\beta}{2}}\right)$ time complexity and $\mathcal{O}\left(2^{\frac{|q|-\beta}{2}}\right)$ space complexity.

Proof. If an attacker learns $s_{A}$, then it knows a HMQV $i$-point for $\hat{A}$ to $\hat{B}$ and its decomposition $\left(X A^{\bar{H}(X, \hat{B})}=G^{s_{A}}\right.$ i.e. $\left.X=G^{s_{A}} A^{-\bar{H}(X, \hat{B})}\right)$; using Attack 2, it impersonates $\hat{A}$ to $\hat{B}$.

\section{Remark 1.}

(a) For the MQV $(-\mathrm{C})$ protocol, if an attacker (partly) learns the ephemeral secret exponent in a session at $\hat{A}$, it can not only impersonate $\hat{A}$ to its peer in the leaked session, but also to any other entity. A weaker form of this attack was proposed in [15].

(b) To meet the two-and-half exponentiations per party performance, which partly makes the attractiveness of the HMQV protocol, the ephemeral secret exponents have to be computed, and the exponentiation $\left(\left(Y B^{e}\right)^{s_{A}}\right.$ or $\left.\left(X A^{e}\right)^{s_{B}}\right)$ performed. Ephemeral secret exponent leakage may happen (through side channel attacks for instance), independently of the ephemeral private keys.

(c) Ephemeral secret exponent leakage does not imply neither static of ephemeral private key discloser. Indeed, one can show that from any algorithm $\mathcal{A}$ with complexity $C_{\mathcal{A}}$, which given $s_{A}, X, A$ and $\hat{B}$, finds $\hat{A}$ 's ephemeral private key $x$ or the static one $a$, one can derive an algorithm which solves two instances of the DLP in $\mathcal{G}^{*}$, in $C_{\mathcal{A}}+C_{D L P}$ time complexity where $C_{D L P}$ is the complexity for solving one instance of the DLP in $\mathcal{G}$.

(d) The leakage of consecutive middle part bits on an ephemeral secret exponent is not discussed, but with tools from [7], the analysis we propose applies in this case with minor modifications.

Ephemeral secret exponent leakage implies (but is not equivalent to) session key reveal, and does not imply neither static key reveal nor ephemeral key reveal; while it is not difficult to see that both ephemeral secret exponent and ephemeral key leakages on the same session imply the session owner's static key discloser.

\subsubsection{Man in the Middle Attack using Session Secret Leakages}

If in addition to $s_{A}$, an attacker learns $s_{B}$ in a session at $\hat{B}$, it can perform man in the middle attacks, between $\hat{A}$ and $\hat{B}$, as in Algorithm 3. We denote by $s_{A}^{(l)}$ and $s_{B}^{(l)}$ the ephemeral secret exponents the attacker learned at $\hat{A}$ and $\hat{B}$ respectively; $X^{(l)}$ and $Y^{(l)}$ are $\hat{A}$ and $\hat{B}$ 's outgoing ephemeral keys in the sessions in which leakages happened. Notice that it is not required that the $\left(s_{A}\right.$ and $\left.s_{B}\right)$ leakages happened in matching sessions.

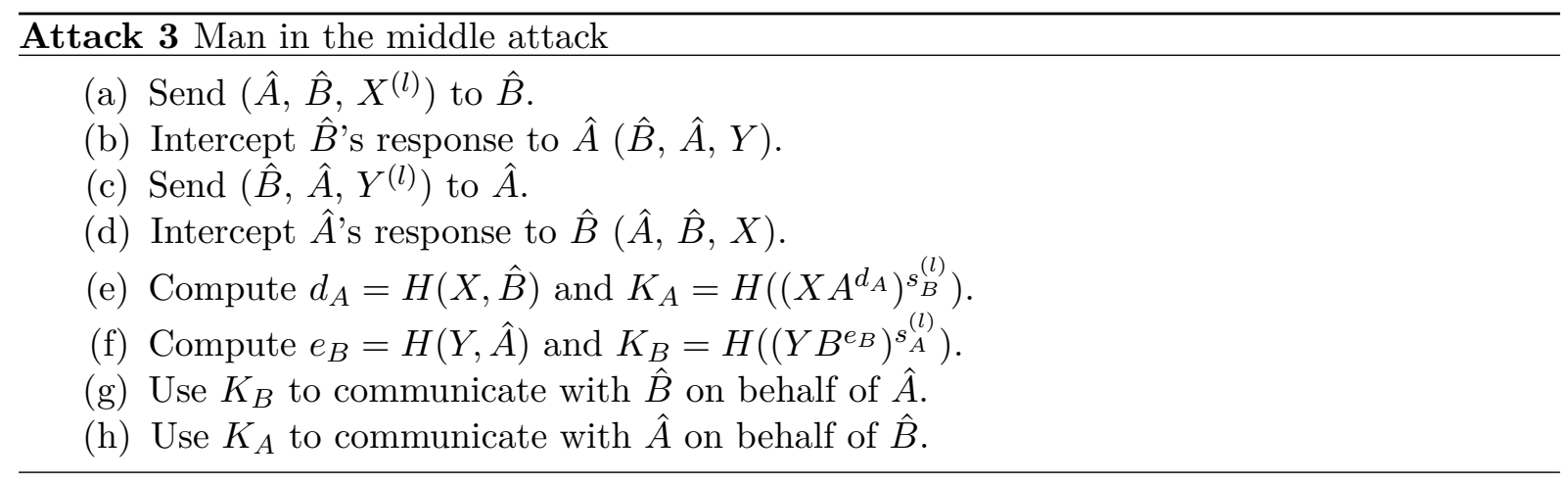

Algorithm 3 is merely a simultaneous impersonation of $\hat{A}$ to $\hat{B}$, and $\hat{B}$ to $\hat{A}$. The session key that $\hat{A}$ derives is $K_{A}=H\left(\left(Y^{(l)} B^{e_{A}}\right)^{x+d_{A} a}\right)=H\left(\left(X A^{d_{A}}\right)^{s_{B}^{(l)}}\right)$, where $e_{A}=H\left(Y^{(l)}, \hat{A}\right)$ and $d_{A}=H(X, \hat{B})$. This is the $K_{A}$ the attacker computes at step (e). Similarly, the session key that $\hat{B}$ derives is $K_{B}=H\left(\left(Y B^{e_{B}}\right)^{s_{A}^{(l)}}\right)$. Notice that the attack remains possible when communications are initiated by $\hat{A}$ (or $\hat{B})$. 


\section{Security Model}

We define a security model, inspired by the (extended) Canetti-Krawczyk model [5, 13], for the (H)MQV type protocols. We aim to a better capture of session specific information leakages. While both ephemeral secret exponent and ephemeral key leakages on the same session imply the session owner's static private key disclosure, resistance to ephemeral secret exponent leakage is a desirable security attribute. We propose a security model which takes into account this security attribute.

Rationale of the model. In the extended Canetti-Krawczyk (eCK) model [13], sessionspecific information leakages are captured through an ephemeral key reveal query. The ephemeral key of a session is required to contain all session specific information. When this requirement is fully satisfied, it becomes difficult to simulate consistently information leakages. In practice, ephemeral keys are not always defined to contain all session specific information; in the NAXOS[13] and CMQV [27] security arguments, ephemeral keys are not defined to contain the ephemeral Diffie-Hellman (DH) exponents.

In addition, session key derivation generally involves some intermediate results, on which leakages may happen; these intermediate results cannot always be computed, given only the session's ephemeral key. Hence leakages on these intermediate results are not necessarily captured in the eCK model. In the CMQV protocol (shown eCK secure) an ephemeral secret exponent leakage, allows an attacker to impersonate indefinitely the leaked session owner.

In the Canetti-Krawczyk (CK) model [5], session secret leakages are captured through a session state reveal query. However, it is not always clear, which information in a session can be revealed, as this is left to be specified by each protocol. Moreover, the ephemeral information that can be available in a session depend on the reached step in the session's computations. Contrary to the ephemeral key reveal query, the session state reveal query cannot be issued on a test session, while it is desirable that an attacker which does not know both the static and ephemeral DH exponents of an entity implicated in a session should not be able to compute the session key. It is also difficult to figure out the practical meaning of the CK-security, as a protocol (HMQV for instance) may be both secure and insecure, depending on the session state's definition.

The ephemeral information that can be available in a session state depends on the reached step in the session's tree of computations. To capture precisely ephemeral information leakages, one has to consider sessions' tree of computations. In the model we propose, the eCK model is completed with reveal queries on sessions intermediate results. We define a reveal query on any intermediate value which computation requires a secret information. With these queries, we aim to an exhaustive capture of ephemeral information leakages. It is however difficult to simultaneously and consistently simulate leakages on both ephemeral keys and intermediate results. This is the reason why our model follows two stages. In the first, leakages on the intermediate results are considered; the second deals with ephemeral private keys leakages.

Session. We suppose $n \leqslant \mathcal{P}(|q|)$ (for some polynomial $\mathcal{P}$ ) parties $\hat{P}_{i, i=1, \ldots, n}$ modeled as probabilistic polynomial time machines, and a certification authority (CA) trusted by all parties. All the static public keys are supposed to belong to $\mathcal{G}^{*}$, this corresponds to the fact that the CA is (only) required to verify that public keys are valid ones. Each party has a static public key together with a certificate binding his identity to his public key.

A session is an instance of a protocol run at a party. A session at $\hat{A}$ (with peer $\hat{B}$ ) can be created with parameter $(\hat{A}, \hat{B})$ or $(\hat{B}, \hat{A}, Y) ; \hat{A}$ is the initiator if the creation parameter is $(\hat{A}, \hat{B})$, otherwise the responder. At session activation, a session state is created to contain the information specific to the session. Each session is identified with a quadruple $(\hat{A}, \hat{B}, X, \star)$, where $\hat{A}$ is the session holder, $\hat{B}$ is the peer, $X$ is the outgoing ephemeral key, and $\star$ is the incoming key $Y$ if it exists, otherwise a special symbol meaning that an incoming ephemeral key is not received yet; in that case when $\hat{A}$ receives the ephemeral public key $Y$, the session identifier 
is updated to $(\hat{A}, \hat{B}, X, Y)$. Two sessions with identifiers $(\hat{B}, \hat{A}, Y, X)$ and $(\hat{A}, \hat{B}, X, Y)$ are said to be matching. Notice that the session matching $(\hat{B}, \hat{A}, Y, X)$ can be any session $(\hat{A}, \hat{B}, X, \star)$; as $X$ and $Y$ are chosen uniformly at random in $\mathcal{G}^{*}$, a session cannot have (except with negligible probability) more than one matching session.

Adversary and Security. The adversary, denoted $\mathcal{A}$, is a probabilistic polynomial time machine. It is a common assumption that an adversary is able to eavesdrop, modify, delete any message sent in a cryptographic protocol, or inject its own messages. This is captured through the assumption that outgoing messages are submitted to $\mathcal{A}$ for delivery $(\mathcal{A}$ decides about messages delivery); $\mathcal{A}$ is also supposed to control session activations at each party $\hat{P}_{i}$ via the $\operatorname{Send}\left(\hat{P}_{i}, \hat{P}_{j}\right)$ and $\operatorname{Send}\left(\hat{P}_{j}, \hat{P}_{i}, Y\right)$ queries, which make $\hat{P}_{i}$ initiate a session with peer $\hat{P}_{j}$ or respond to $\hat{P}_{j}$.

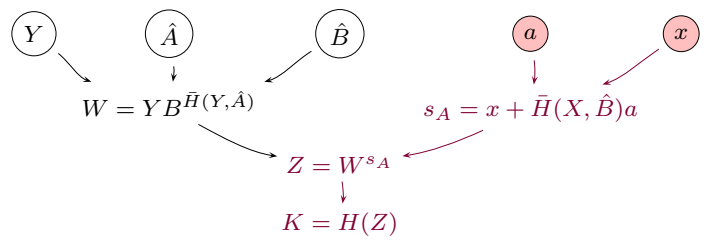

Figure 1: Tree of computations in a HMQV session.

The adversary is also provided with the reveal queries from one of the following sets. At the beginning of its run, it adopts one of the following sets of queries; it can then perform queries from the selected set (and only those queries).

In Set 1, the following queries are allowed.

- StaticKeyReveal(party) to obtain the static private key of a party.

- SessionKeyReveal(session) to obtain the derived key in a session.

- SecretExponentReveal(session) to obtain the ephemeral secret exponent ( $s=x+d a$ or $y+e b)$ in a session.

- SecretGroupElementReveal(session) to obtain the session signature $Z=W^{s_{A}}$.

- EstablishParty(party) to register a static public key on behalf of a party; from there, the party is supposed totally controlled by $\mathcal{A}$. A party against which this query is not issued is said to be honest.

Notice that, we consider only the intermediate values which evaluation requires a secret information; as the attacker is supposed to control the communication links between parties, considering leakages on the other intermediate values is superfluous. We also implicitly assume that the considered protocol has a tree of computations "matching" that of the $(\mathrm{H}) \mathrm{MQV}$ protocols; otherwise some queries (SecretExponentReveal for instance) may become meaningless.

In Set 2, the allowed queries are the following; the definitions remain unchanged for the queries belonging also to Set 1 .

- EphemeralKeyReveal(session) to obtain the ephemeral private key used by the session owner.

- StaticKeyReveal(party).

- SessionKeyReveal(session).

- EstablishParty(party).

Definition 4 (Session Freshness). Let sid be the identifier of a completed session at an honest party $\hat{A}$, with some honest peer $\hat{B}$, and $s^{*} d^{*}$ the matching session's identifier. The session sid is said to be $c k-f r e s h$, if none of the following conditions hold:

- $\mathcal{A}$ issues a SecretExponentReveal query on sid or sid* (if sid* exists);

- $\mathcal{A}$ issues a SecretGroupElementReveal query on sid or sid*;

- $\mathcal{A}$ issues a SessionKeyReveal query on sid or sid*;

- sid* does not exist and $\mathcal{A}$ makes a StaticKeyReveal query on $\hat{B}$.

And sid is said to be eck-fresh, if none of the following conditions hold:

- $\mathcal{A}$ issues a SessionKeyReveal query on sid or sid* (if sid $^{*}$ exists); 
- $\mathcal{A}$ issues a StaticKeyReveal query on $\hat{A}$ and an EphemeralKeyReveal query on sid;

- sid* exists and $\mathcal{A}$ makes a StaticKeyReveal query on $\hat{B}$ and an EphemeralKeyReveal query on sid $^{*}$;

- sid $^{*}$ does not exist and $\mathcal{A}$ makes a StaticKeyReveal query on $\hat{B}$.

Definition 5 (Protocol Security). Let $\Pi$ be a protocol, such that if two honest parties complete matching sessions, then they both compute the same session key.

- The protocol $\Pi$ is said to be $c k-$ secure, if no polynomially bounded adversary (performing queries from Set 1) can distinguish a ck-fresh session key from a random value, chosen under the distribution of session keys, with probability (taken over the random coins of the adversary and the choices of static and ephemeral public keys in $\mathcal{G}$ ) significantly greater than $1 / 2$.

- $\Pi$ is said to be eck-secure, if no polynomially bounded adversary (performing queries from Set 2) can distinguish an eck-fresh session key from a random value, chosen under the distribution of session keys, with probability significantly greater than $1 / 2$.

- And $\Pi$ is said to be secure, if it is both ck-secure and eck-secure.

\section{A New Authenticated Diffie-Hellman Protocol}

In this section, we define the Full Exponential Challenge Response (FXCR) and Full Dual exponential Challenge Response (FDCR) schemes, which confine to the minimum the consequences of ephemeral secret exponent leakages. Using these schemes, we define the Fully Hashed MQV (FHMQV) protocol, which preserves the performance of the (H)MQV protocol, in addition to provide resistance to the attacks we presented in section 2 .

\subsection{Full Exponential Challenge Response Signature Scheme}

Definition 6 (FXCR signature scheme). Let $\hat{B}$ be an entity with public key $B \in \mathcal{G}^{*}$, and $\hat{A}$ a verifier. $\hat{B}$ 's signature on a message $m$ and challenge $X$ provided by $\hat{A}\left(X=G^{x}, x \in_{R}[1, q-1]\right.$ is chosen and kept secret by $\hat{A})$ is $F \operatorname{Sig}_{\hat{B}}(m, X)=\left(Y, X^{s_{B}}\right)$, where $Y=G^{y}, y \in_{R}[1, q-1]$ is chosen by $\hat{B}$, and $s_{B}=y+\bar{H}(Y, X, m) b$; the verifier $\hat{A}$ accepts the pair $\left(Y, \sigma_{B}\right)$ as a valid signature if $Y \in \mathcal{G}^{*}$ and $\left(Y B^{\bar{H}(Y, X, m)}\right)^{x}=\sigma_{B}$.

The FXCR scheme delivers all the security attributes of the XCR scheme; in addition the "replay attack" we presented in section 2 does not hold anymore. Indeed, suppose an attacker which has learned $s_{B}{ }^{(l)}=y^{(l)}+\bar{H}\left(Y^{(l)}, X^{(l)}, m\right) b$. When it is provided with a new challenge $X$ (chosen at random) and the same message $m$, except with negligible probability $X \neq X^{(l)}$ (and $\left.\bar{H}\left(Y^{(l)}, X^{(l)}, m\right) \neq \bar{H}\left(Y^{(l)}, X, m\right)\right)$. Hence, to replay $Y^{(l)}$ on the message-challenge pair $(m, X)$, the attacker has to find $s_{B}=y^{(l)}+\bar{H}\left(Y^{(l)}, X, m\right) b$; it is not difficult to see that if it can compute $s_{B}$ from $s_{B}{ }^{(l)}$, then he can find $b$ from $s_{B}$, which is not feasible.

Definition 7 (FXCR Scheme Security). The FXCR scheme is said to be secure in $\mathcal{G}$, if given a public key $B$, a challenge $X_{0}\left(B, X_{0} \in_{R} \mathcal{G}^{*}\right)$, together with hashing and signing oracles, no adaptive probabilistic polynomial time attacker, can output with non negligible success probability a triple $\left(m_{0}, Y_{0}, \sigma_{0}\right)$ such that:

- $\left(Y_{0}, \sigma_{0}\right)$ is a valid signature with respect to the key $B$, and the message-challenge pair $\left(m_{0}, X_{0}\right)$

- $\left(Y_{0}, \sigma_{0}\right)$ was not obtained from the signing oracle with a query on $\left(m_{0}, X_{0}\right)$.

Using the "oracle replay" technique $[22,23]$, we show that the FXCR scheme is secure in the sense of definition 7 . Recall that a function $\mathcal{F}$ with parameter $\zeta$ is said to be negligible, if for every polynomial $\mathcal{P}$, and every sufficiently large $\zeta, \mathcal{F}(\zeta)<(\|\mathcal{P}(\zeta)\|)^{-1}(\|\cdot\|$ denotes here the absolute value); otherwise $\mathcal{F}$ is said to be non-negligible.

Proposition 4. Under the $C D H$ assumption in $\mathcal{G}$ and the $R O$ model, the FXCR signature scheme is secure in the sense of definition $\%$. 
Proof. Suppose an attacker $\mathcal{A}$, which given $B, X_{0} \in_{R} \mathcal{G}^{*}$ succeeds with non-negligible probability in forging a FXCR signature, with respect to the public key $B$ and the challenge $X_{0}$. Using $\mathcal{A}$ we build a polynomial time $\mathrm{CDH}$ solver $\mathcal{S}$ which succeeds with non-negligible probability. The solver $\mathcal{S}$ provides $\mathcal{A}$ with random coins, and simulates the digest and signature queries. The interactions between $\mathcal{S}$ and $\mathcal{A}$ are described in Figure 4.

Figure $4 \mathrm{~A}$ CDH solver from $\mathcal{A}$

Run of $\mathcal{A}$ :

(a) At $\mathcal{A}$ 's digest query on $(Y, X, m), \mathcal{S}$ responds as follows: (i) if a value is already assigned to $\bar{H}(Y, X, m), \mathcal{S}$ returns $\bar{H}(Y, X, m)$; (ii) otherwise $\mathcal{S}$ responds with $e \in_{R}\{0,1\}^{l}$, and sets $\bar{H}(Y, X, m)=e$.

(b) At $\mathcal{A}$ 's signature query on $(m, X), \mathcal{S}$ responds as follows: (i) $\mathcal{S}$ chooses $s_{B} \in_{R}[1, q-1]$, $e \in_{R}\{0,1\}^{l}$, sets $Y=G^{s_{B}} B^{-e}$ and $\bar{H}(Y, X, m)=e$. If $\bar{H}(Y, X, m)$ was previously defined, $\mathcal{S}$ aborts. (ii) Else, $\mathcal{S}$ responds with $\left(Y, X^{s_{B}}\right)$.

(c) At $\mathcal{A}$ 's halt, $\mathcal{S}$ verifies that $\mathcal{A}$ 's output $\left(m_{0}, Y_{0}, \sigma_{0}^{(1)}\right)$ (if any) satisfies the following conditions. If not, $\mathcal{S}$ aborts.

- $Y_{0} \in \mathcal{G}^{*}$ and $\bar{H}\left(Y_{0}, X_{0}, m_{0}\right)$ was queried from $\bar{H}$.

- $\left(Y_{0}, \sigma_{0}^{(1)}\right)$ was not returned by $\hat{B}$ at signature query on $\left(m_{0}, X_{0}\right)$.

Repeat: $\mathcal{S}$ executes a new run of $\mathcal{A}$, using the same input and coins; and answering to all the digest queries before $\bar{H}\left(Y_{0}, X_{0}, m_{0}\right)$ with the same values as in the previous run. The new query of $\bar{H}\left(Y_{0}, X_{0}, m_{0}\right)$ and subsequent $\bar{H}$ queries are answered with new random values.

Output: If $\mathcal{A}$ outputs a second forgery $\left(m_{0}, Y_{0}, \sigma_{0}^{(2)}\right)$ satisfying the conditions of step (c), with a hash value $\bar{H}\left(Y_{0}, X_{0}, m_{0}\right)_{2}=e_{0}^{(2)} \neq e_{0}^{(1)}=\bar{H}\left(Y_{0}, X_{0}, m_{0}\right)_{1}$, then $\mathcal{S}$ outputs $\left(\sigma_{0}^{(1)} / \sigma_{0}^{(2)}\right)^{\left(e_{0}^{(1)}-e_{0}^{(2)}\right)^{-1}}$ as a guess for $C D H\left(B, X_{0}\right)$.

Under the RO model, the distribution of the simulated signatures is indistinguishable from the that of the real signatures generated by $\hat{B}$, except the deviation that happens when $\bar{H}(Y, X, m)$ was queried before. Let $Q_{h}$ and $Q_{s}$ be respectively the number of queries that $\mathcal{A}$ asks to the hashing and signing oracles. Since $\mathcal{A}$ is polynomial (in $|q|$ ) and $Y$ is chosen uniformly at random in $\mathcal{G}$, this deviation happens with probability less than $\left(Q_{h}+Q_{s}\right) / q$, which is negligible. Hence this simulation is perfect, except with negligible probability. Moreover the probability of producing a valid forgery without querying $\bar{H}\left(Y_{0}, X_{0}, m_{0}\right)$ is $2^{-l}$. Thus under this simulation, $\mathcal{A}$ outputs with non-negligible probability a valid forgery $\left(m_{0}, Y_{0}, \sigma_{0}^{(1)}\right)$; we denote $\bar{H}\left(Y_{0}, X_{0}, m_{0}\right)$ by $e_{0}^{(1)}$. From the Forking lemma [23], the repeat experiment produces with non-negligible probability a valid forgery $\left(m_{0}, Y_{0}, \sigma_{0}^{(2)}\right)$ with a digest $e_{0}^{(2)}$, which with probability $1-2^{-l}$, is different from $e_{0}^{(1)}$. Then the computation

$$
\left(\frac{\sigma_{0}^{(1)}}{\sigma_{0}^{(2)}}\right)^{\left(e_{0}^{(1)}-e_{0}^{(2)}\right)^{-1}}=\left(\frac{\left(Y_{0} B^{e_{0}^{(1)}}\right)^{x_{0}}}{\left(Y_{0} B^{e_{0}^{(2)}}\right)^{x_{0}}}\right)^{\left(e_{0}^{(1)}-e_{0}^{(2)}\right)^{-1}}=B^{x_{0}}
$$

gives $C D H\left(B, X_{0}\right)$ with non-negligible success probability.

\subsection{Full Dual Exponential Challenge Response Signature Scheme}

Definition 8 (FDCR Signature Scheme). Let $\hat{A}$ and $\hat{B}$ be two entities with public keys $A, B \in \mathcal{G}^{*}$, and $m_{1}, m_{2}$ two messages. The FDCR signature of $\hat{A}$ and $\hat{B}$ on messages $m_{1}, m_{2}$ is $F D$ Sig $_{\hat{A}, \hat{B}}\left(m_{1}, m_{2}, X, Y\right)=\left(X A^{d}\right)^{y+e b}=\left(Y B^{e}\right)^{x+d a}$, where $X=G^{x}$ and $Y=G^{y}\left(x, y \in_{R}\right.$ $[1, q-1])$ are chosen respectively by $\hat{A}$ and $\hat{B}, d=\bar{H}\left(X, Y, m_{1}, m_{2}\right)$, and $e=\bar{H}\left(Y, X, m_{1}, m_{2}\right)$.

Notice that contrary to the DCR and XCR schemes, the FDCR signature of $\hat{A}$ and $\hat{B}$ on messages $m_{1}, m_{2}$ and challenges $X, Y$, is not a FXCR signature of $\hat{A}$ on the message $m_{1}$ and challenge $Y B^{e}$. 
Definition 9 (Security of the FDCR Scheme). Let $A=G^{a}, B, X_{0} \in_{R} \mathcal{G}^{*}(A \neq B)$. The FDCR scheme is secure in $\mathcal{G}$, if given $a, A, B, X_{0}$, and a message $m_{1_{0}}$, together with hashing and signing oracles, no adaptive probabilistic polynomial time attacker can output with non negligible success probability a triple $\left(m_{2_{0}}, Y_{0}, \sigma_{0}\right)$ such that:

- $\left(m_{1_{0}}, m_{2_{0}}, X_{0}, Y_{0}, \sigma_{0}\right)$ is a valid FDCR signature with respect to the public keys $A, B$.

- $\left(Y_{0}, \sigma_{0}\right)$ was not obtained from the signing oracle with a query on a message-challenge pair $\left(m_{1}^{\prime}, X_{0}\right)$ such that $\left(m_{1}^{\prime}, m_{2}^{\prime}\right)=\left(m_{1_{0}}, m_{2_{0}}\right)$, where $\left(m_{1_{0}}, m_{2_{0}}\right)$ denotes the concatenation of $m_{1_{0}}$ and $m_{2_{0}}$, and $m_{2}^{\prime}$ is the message returned at signature query on $\left(m_{1}^{\prime}, X_{0}\right)$ (if any).

Remark 2. Since we suppose that if $\hat{A} \neq \hat{A}^{\prime}$, no substring of $\hat{A}$ equals $\hat{A}^{\prime}$ (and conversely), if $\hat{A} \neq \hat{A}^{\prime}$ or $\hat{B} \neq \hat{B}^{\prime}$ then $(\hat{A}, \hat{B})$ cannot equal $\left(\hat{A}^{\prime}, \hat{B}^{\prime}\right)$.

Proposition 5. Under the RO model, and the CDH assumption in $\mathcal{G}$, the FDCR scheme is secure in the sense of Definition 9.

Proof. Suppose an attacker $\mathcal{A}$, which given $a, A, B, X_{0}, m_{1_{0}}(A \neq B)$ outputs with non-negligible probability a valid and fresh FDCR forgery $\left(m_{2_{0}}, Y_{0}, \sigma_{0}\right)$. Using $\mathcal{A}$ we build a polynomial time FXCR forger which succeeds with non-negligible probability. The FXCR forger $\mathcal{S}$ provides $\mathcal{A}$ with random coins, $a, A, B, X_{0}, m_{1_{0}}$, and simulates $\hat{B}$ 's role as follows.

(a) At $\mathcal{A}$ 's digest query on $\left(X, Y, m_{1}, m_{2}\right), \mathcal{S}$ responds as follows: (i) if a value is already assigned to $\bar{H}\left(X, Y, m_{1}, m_{2}\right), \mathcal{S}$ returns $\bar{H}\left(X, Y, m_{1}, m_{2}\right)$; (ii) else $\mathcal{S}$ responds with $d \in_{R}\{0,1\}^{l}$, and sets $\bar{H}\left(X, Y, m_{1}, m_{2}\right)=d$.

(b) At signature query on $\left(m_{1}, X\right), \mathcal{S}$ responds as follows: (i) $\mathcal{S}$ chooses $m_{2} \in_{R}\{0,1\}^{*}$, $s_{B} \in_{R}[1, q-1], d, e \in_{R}\{0,1\}^{l}$, computes $Y=G^{s_{B}} B^{-e}$, and sets $\bar{H}\left(X, Y, m_{1}, m_{2}\right)=d$ and $\bar{H}\left(Y, X, m_{1}, m_{2}\right)=e$; if $\bar{H}\left(X, Y, m_{1}, m_{2}\right)$ or $\bar{H}\left(Y, X, m_{1}, m_{2}\right)$ was previously defined, $\mathcal{S}$ aborts. (ii) $\mathcal{S}$ provides $\mathcal{A}$ with the signature $\left(m_{2}, Y,\left(X A^{d}\right)^{s_{B}}\right)$.

The simulated environment is perfect, except with negligible probability. The deviation happens when the same message-challenge pair $\left(m_{2}, Y\right)$ is chosen twice in two signature queries on the same pair $\left(m_{1}, X\right)$. Let $Q_{\max }$ is the maximum number of queries that $\mathcal{A}$ asks to the signing oracle. Since $Y$ is chosen uniformly at random in $\mathcal{G}$ and $\mathcal{A}$ is polynomial, the deviation occurs with probability less than $Q_{\max } / q$, which is negligible (as $\mathcal{A}$ is polynomial in $|q|$.) Then, if $\mathcal{A}$ succeeds with non-negligible probability in FDCR forging attack, it succeeds also with nonnegligible probability under this simulation. And since $\mathcal{S}$ knows $a$, it outputs, from any valid forgery $\sigma_{0}$,

$$
\sigma_{0}\left(Y_{0} B^{e}\right)^{-d a}=\left(Y_{0} B^{e}\right)^{x_{0}+d a}\left(Y_{0} B^{e}\right)^{-d a}=X_{0}^{y_{0}+e b} .
$$

This is valid FXCR forgery on the message $\left(m_{1_{0}}, m_{2_{0}}\right)$ (the concatenation of $m_{1_{0}}$ and $m_{2_{0}}$ ) and challenge $X_{0}$ with respect to the public key $B$. And if $\mathcal{A}$ succeeds with non-negligible probability, so does $\mathcal{S}$, contradicting Proposition 4 .

\subsection{The Fully Hashed MQV Protocol.}

We can now derive the FHMQV protocol, which provides the efficiency and security attributes of the $(\mathrm{H}) \mathrm{MQV}$ protocols, in addition to ephemeral secret exponent leakage resilience.

The FHMQV protocol does not only provide a stronger security than the $(\mathrm{C}, \mathrm{H}) \mathrm{MQV}$ protocols; it seems more suited for implementations using computationally limited devices, to store (and protect) the private keys. Suppose an implementation of FHMQV or (H)MQV (using such devices) in which session keys are used by some application running in a untrusted host machine. Suppose that the ephemeral keys are computed in the device in idle-time. This idle-time pre-computation is common in practice. As $(\mathrm{H}) \mathrm{MQV}$ is not ephemeral secret exponent leakage resilient, the exponentiation $\sigma=\left(Y B^{e}\right)^{s_{A}}=\left(X A^{d}\right)^{s_{B}}$ has to be performed in the device (in non idle-time). In contrast, for FHMQV $\sigma$ can be computed in the host machine, after the ephemeral secret exponent $\left(s_{A}\right.$ or $\left.s_{B}\right)$ is computed in the device. (Because the session key is 
I) The initiator $\hat{A}$ does the following:

(a) Choose $x \in_{R}[1, q-1]$ and compute $X=G^{x}$.

(b) Send $(\hat{A}, \hat{B}, X)$ to $\hat{B}$.

II) At receipt of $(\hat{A}, \hat{B}, X), \hat{B}$ does the following:

(a) Verify that $X \in \mathcal{G}^{*}$.

(b) Choose $y \in_{R}[1, q-1]$ and compute $Y=G^{y}$.

(c) Send $(\hat{B}, \hat{A}, Y)$ to $\hat{A}$.

(d) Compute $d=\bar{H}(X, Y, \hat{A}, \hat{B})$ and $e=\bar{H}(Y, X, \hat{A}, \hat{B})$.

(e) Compute $s_{B}=y+e b \bmod q, \sigma_{B}=\left(X A^{d}\right)^{s_{B}}$, and $K=H\left(\sigma_{B}, \hat{A}, \hat{B}, X, Y\right)$.

III) At receipt of $(\hat{B}, \hat{A}, Y), \hat{A}$ does the following:

(a) Verify that $Y \in \mathcal{G}^{*}$.

(b) Compute $d=\bar{H}(X, Y, \hat{A}, \hat{B})$ and $e=\bar{H}(Y, X, \hat{A}, \hat{B})$.

(c) Compute $s_{A}=x+d a \bmod q, \sigma_{A}=\left(Y B^{e}\right)^{s_{A}}$, and $K=H\left(\sigma_{A}, \hat{A}, \hat{B}, X, Y\right)$.

IV) The shared session key is $K$.

used in the host machine, and a leakage of only the ephemeral secret exponent, in a FHMQV session, does not compromise any other session.)

In FHMQV the computational effort of the device, in non idle-time can be safely reduced to few non-costly operations (two integer additions, one integer multiplication, and two digest computations), while for (H)MQV at least one exponentiation has to be performed in the device in non idle-time (in addition to few non-costly operations).

\section{Security Analysis of the FHMQV Protocol}

We suppose $n \leqslant \mathcal{P}(|q|)$ (for some polynomial $\mathcal{P}$ ) parties modeled as probabilistic polynomial time machines. In accord with our security model, the following queries are allowed.

- $\operatorname{Send}(\hat{A}, \hat{B})$ which makes $\hat{A}$ perform the step I of Protocol 5.

- $\operatorname{Send}(\hat{A}, \hat{B}, X)$ which makes $\hat{B}$ perform the step II of Protocol 5.

- $\operatorname{Send}(\hat{A}, \hat{B}, X, Y)$ which makes $\hat{A}$ update the session identifier $(\hat{A}, \hat{B}, X, \star)$ (if any) to $(\hat{A}, \hat{B}, X, Y)$, and perform the step III of Protocol 5 ; if $\hat{A}$ does not hold a session with identifier $(\hat{A}, \hat{B}, X, \star)$, the call is ignored.

\subsection{Ck-Security Arguments}

Proposition 6. Under the CDH assumption in $\mathcal{G}$, and the RO model, the FHMQV protocol is ck-secure.

It is immediate from the FHMQV definition that, if two honest parties complete matching sessions, then they both compute the same session key. Suppose an adversary $\mathcal{A}$ which succeeds, with probability significantly greater than $1 / 2$, in distinguishing a session key of a ck-fresh session (that we designate by $\left(\hat{A}, \hat{B}, X_{0}, Y_{0}\right)$ or test session) from random a value chosen under the distribution of session keys. $\mathcal{A}$ can only distinguish a ck-fresh session key from a random value in one of the following ways.

Guessing attack: $\mathcal{A}$ guesses correctly the test session key.

Key replication attack: $\mathcal{A}$ succeeds in making two non-matching sessions yield the same session key, it can then query a session key reveal on one of the two sessions and use the other as test session.

Forging attack: $\mathcal{A}$ computes the test session signature and issues digest query to compute the session key. 
Under the RO model, the probability of guessing correctly the output of the hash function is $2^{-k}$; and non-matching sessions cannot have (except with negligible probability) the same session key. It thus remains that if $\mathcal{A}$ succeeds with probability significantly greater than $1 / 2$ in distinguishing a ck-fresh session key, from a random value chosen under the distribution of session keys, then it succeeds with non-negligible probability in forging attack. We thus suppose that $\mathcal{A}$ interacts in an $n$ parties environment, and ends its run with non-negligible probability with an output $\left(\operatorname{sid}_{0}, \sigma_{0}\right)$, where $\operatorname{sid}_{0}$ is a ck-fresh session identifier, and $\sigma_{0}$ a guess of the $s i d_{0}$ session signature.

Let $E$ denote the event " $\mathcal{A}$ succeeds in forging the session signature of some ck-fresh session." The event $E$ divides in: (a) E.1: " $\mathcal{A}$ succeeds in forging the session signature of some ck-fresh session, which matching session exits", and (b) E.2: "A succeeds in forging the session signature of some ck-fresh session without matching session." Since a session is required to be ck-fresh, in E.1 the StaticKeyReveal query is allowed on both $\hat{A}$ and $\hat{B}$. In E.2 the StaticKeyReveal query is allowed on $\hat{A}$ (but not on $\hat{B}$ ). Now since $\mathcal{A}$ succeeds with non-negligible probability, either $E .1$ or E.2 occurs with non-negligible probability; it then suffices to show that neither E.1 nor E.2 can happen with non-negligible probability.

\subsubsection{Analysis of E.1}

Suppose that E.1 occurs with non-negligible probability. Using $\mathcal{A}$, we build a polynomial time $\mathrm{CDH}$ solver which succeeds with non-negligible probability. The solver $\mathcal{S}$ takes as input $X_{0}, Y_{0} \in_{R} \mathcal{G}^{*}$ and interacts with $\mathcal{A}$ as described below.

(1) $\mathcal{S}$ simulates $\mathcal{A}$ 's environment, with $n$ parties $\hat{P}_{1}, \ldots, \hat{P}_{n}$; recall that $\mathcal{A}$ is supposed to be polynomial, we thus suppose that each party is activated at most $m$ times, $m \leqslant \mathcal{P}(|q|)$ for some polynomial $\mathcal{P}$.

(2) $\mathcal{S}$ chooses $i, j \in_{R}\{1, \ldots, n\}, i \neq j$ (the case $i=j$, reflection attack, is analyzed in subsection 5.1.3), and $t \in_{R}\{1, \ldots, m\}$ (with the choice of $(i, j, t), \mathcal{S}$ is guessing the test session). We refer to $\hat{P}_{i}$ as $\hat{A}$ and $\hat{P}_{j}$ as $\hat{B}$. $\mathcal{S}$ assigns to each $\hat{P}_{k}$ a random static key pair $\left(p_{k}, P_{k}=G^{p_{k}}\right)$, and answers to $\mathcal{A}$ queries as follows; $\bar{H}$ queries are simulated in the usual way (see the proof of Proposition 5).

(3) At $\mathcal{A}$ 's $\operatorname{Send}\left(\hat{P}_{l}, \hat{P}_{m}\right)$ query, $\mathcal{S}$ chooses $x \in_{R}[1, q-1]$, computes $X=G^{x}$, creates a session state with identifier $\left(\hat{P}_{l}, \hat{P}_{m}, X, \star\right)$, and provides $\mathcal{A}$ with the message $\left(\hat{P}_{l}, \hat{P}_{m}, X\right)$.

(4) At $\mathcal{A}$ 's $\operatorname{Send}\left(\hat{P}_{m}, \hat{P}_{l}, Y\right) \mathcal{S}$ chooses $x \in_{R}[1, q-1]$, computes $X=G^{x}$, creates a session state with identifier $\left(\hat{P}_{l}, \hat{P}_{m}, X, Y\right)$, provides $\mathcal{A}$ with the message $\left(\hat{P}_{l}, \hat{P}_{m}, X\right)$; and completes the session with identifier $\left(\hat{P}_{l}, \hat{P}_{m}, X, Y\right)$ as responder (step IIe of Protocol 5).

(5) At $\mathcal{A}$ 's $\operatorname{Send}\left(\hat{P}_{l}, \hat{P}_{m}, X, Y\right)$ query, $\mathcal{S}$ updates the session identifier $\left(\hat{P}_{l}, \hat{P}_{m}, X, \star\right)$ to $\left(\hat{P}_{l}, \hat{P}_{m}, X, Y\right)$; and completes the session with identifier $\left(\hat{P}_{l}, \hat{P}_{m}, X, Y\right)$ as initiator (step IIIc of Protocol 5$)$. (Notice that $\mathcal{S}$ can compute the session FDCR signature of any session different from the $t$-th session at $\hat{A}$ - and its matching session.)

(6) When $\mathcal{A}$ activates the $t$-th session at $\hat{A}$, if the peer is $\hat{B}, \mathcal{S}$ provides $\mathcal{A}$ with the message $\left(\hat{A}, \hat{B}, X_{0}\right)$; otherwise, $\mathcal{S}$ aborts.

(7) When $\mathcal{A}$ activates the session matching $t$-th session at $\hat{A}, \mathcal{S}$ provides $\mathcal{A}$ with $\left(\hat{B}, \hat{A}, Y_{0}\right)$.

(8) At $\mathcal{A}$ 's digest query on $\left(\sigma, \hat{P}_{l}, \hat{P}_{m}, X, Y\right), \mathcal{S}$ responds as follows:

- If there exists a completed session with identifier sid $=\left(\hat{P}_{l}, \hat{P}_{m}, X, Y\right)$ or sid $=\left(\hat{P}_{m}, \hat{P}_{l}, Y, X\right)$ and with initiator $\hat{P}_{l}$, and if $\sigma$ is the sid session's FDCR signature, $\mathcal{S}$ returns the sid session key.

- Else, if the same query was made previously, $\mathcal{S}$ returns the previously returned value.

- Else $\mathcal{S}$ responds with $\pi \in_{R}\{0,1\}^{\lambda}$, and sets $H\left(\sigma, \hat{P}_{l}, \hat{P}_{m}, X, Y\right)=\pi$.

(9) If $\mathcal{A}$ issues a StaticKeyReveal, SecretExponentReveal, SecretGroupElementReveal, SessionKeyReveal, or an EstablishParty query, $\mathcal{S}$ answers faithfully.

(10) In any of the following situations, $\mathcal{S}$ aborts.

- $\mathcal{A}$ halts with a test session different from the $t$-th session at $\hat{A}$.

- $\mathcal{A}$ issues a SecretExponentReveal, a SecretGroupElementReveal, or a SessionKeyReveal 
query on the $t$-th session at $\hat{A}$ or its matching session.

- $\mathcal{A}$ issues an EstablishParty query on $\hat{A}$ or $\hat{B}$.

(11) If $\hat{A}$ provides a guess $\sigma_{0}$ of the signature of the $t$-th session at $\hat{A}, \mathcal{S}$ outputs $\left(\sigma_{0}\right)\left(X_{0} A^{d}\right)^{-e b} Y_{0}^{-d a}$ as a guess for $C D H\left(X_{0}, Y_{0}\right)$. Otherwise $\mathcal{S}$ aborts.

Fact. If $\mathcal{A}$ succeeds with non-negligible probability in E.1, $\mathcal{S}$ outputs with non-negligible probability $C D H\left(X_{0}, Y_{0}\right)$.

Proof. The simulation of $\mathcal{A}$ 's environment is perfect except with negligible probability; when $\mathcal{A}$ activates the $t$-th session at $\hat{A}$, the $X_{0}$ provided to $\mathcal{A}$ is chosen uniformly at random in $\mathcal{G}^{*}$, its distribution is the same as that of the real $X$. The same argument holds for $Y_{0}$. The probability of guessing correctly the test session is $\left(n^{2} m\right)^{-1}$; and if $E .1$ occurs and $\mathcal{S}$ guesses correctly the test session, $\mathcal{S}$ does not abort. Thus $\mathcal{S}$ succeeds with probability $\left(n^{2} m\right)^{-1} \operatorname{Pr}(E .1)$ where negligible terms are ignored. In addition, when $\mathcal{A}$ outputs a correct guess for the test session signature, $\mathcal{S}$ outputs

$$
\left(\sigma_{0}\right)\left(X_{0} A^{d}\right)^{-e a} Y_{0}^{-d a}=\left(X_{0} A^{d}\right)^{y_{0}+a e}\left(X_{0} A^{d}\right)^{-e a} Y_{0}^{-d a}=Y_{0}^{x_{0}+d a} Y_{0}^{-d a}=C D H\left(X_{0}, Y_{0}\right) .
$$

Moreover if $\mathcal{A}$ is polynomial, $\mathcal{S}$ is also polynomial. This shows that E.1 cannot happen with non-negligible probability.

\subsubsection{Analysis of E.2}

If E.2 occurs with non-negligible probability, using $\mathcal{A}$, we build a polynomial time FDCR forger, with non-negligible success probability. For this purpose, we modify the simulation in the analysis of $E .1$ as follows.

- $\mathcal{S}$ takes as input $a \in_{R}[1, q-1]$, and $X_{0}, B \in_{R} \mathcal{G}^{*}$.

- $\hat{A}$ 's key pair is set to $\left(a, G^{a}\right)$, and $\hat{B}$ 's public key to $B$ ( $\hat{B}$ 's private key is unknown to $\mathcal{S}$ ).

- At $\mathcal{A}$ 's $\operatorname{Send}\left(\hat{P}_{l}, \hat{B}, X\right)$ query, $\mathcal{S}$ answers as follows:

$-\mathcal{S}$ chooses $s_{B} \in_{R}[1, q-1], d, e \in_{R}\{0,1\}^{l}$, and sets $Y=G^{s_{B}} B^{-e}, \bar{H}\left(X, Y, \hat{P}_{l}, \hat{B}\right)=d$, and $\bar{H}\left(Y, X, \hat{P}_{l}, \hat{B}\right)=e$; if $Y$ was previously used as outgoing ephemeral key in a session at $\hat{B}$ with peer $\hat{P}_{l}, \mathcal{S}$ aborts.

- $\mathcal{S}$ creates a session state with identifier $\left(\hat{B}, \hat{P}_{l}, Y, X\right)$, and provides $\mathcal{A}$ with $\left(\hat{B}_{1}, \hat{P}_{l}, Y\right)$.

- $\mathcal{S}$ completes the session $\left(\hat{B}, \hat{P}_{l}, Y, X\right)$ as responder.

- At $\mathcal{A}$ 's $\operatorname{Send}\left(\hat{B}, \hat{P}_{l}\right)$ query:

$-\mathcal{S}$ chooses $s_{B} \in_{R}[1, q-1], d, e \in \in_{R}\{0,1\}^{l}$, and $\operatorname{sets}^{3} Y=G^{s_{B}} B^{-e}, \bar{H}\left(\star, Y, \hat{B}, \hat{P}_{l}\right)=d$, $\bar{H}\left(Y, \star, \hat{B}, \hat{P}_{l}\right)=e$; if $Y$ was previously used as outgoing ephemeral key in a session at $\hat{B}$ with peer $\hat{P}_{l}, \mathcal{S}$ aborts.

- $\mathcal{S}$ creates a session state with identifier $\left(\hat{B}, \hat{P}_{l}, Y, \star\right)$, and provides $\mathcal{A}$ with the message $\left(\hat{B}, \hat{P}_{l}, Y\right)$.

- When $\mathcal{A}$ activates the $t$-th session at $\hat{A}$, if the peer is not $\hat{B}, \mathcal{S}$ aborts; else, $\mathcal{S}$ provides $\mathcal{A}$ with $\left(\hat{A}, \hat{B}, X_{0}\right)$.

- $\mathcal{S}$ aborts in any of the following situations.

$-\mathcal{A}$ activates at $\hat{B}$ a session matching the $t$-th session at $\hat{A}$.

- $\mathcal{A}$ halts with a test session different from the $t$-th session at $\hat{A}$.

- $\mathcal{A}$ issues a StaticKeyReveal query on $\hat{B}$, or an EstablishParty query on $\hat{A}$ or $\hat{B}$.

- $\mathcal{A}$ issues a SecretExponentReveal, SessionSignatureReveal, or a SessionKeyReveal query on the $t$-th session at $\hat{A}$.

- If $\hat{A}$ halts with the $t$-th session at $\hat{A},\left(\hat{A}, \hat{B}, X_{0}, Y_{0}\right)$ as test session, and with a guess $\sigma_{0}$ of the session's signature, $\mathcal{S}$ outputs the triple $\left((\hat{A}, \hat{B}), Y_{0}, \sigma_{0}\right)$ as a FDCR forgery on messages $\hat{A}, \hat{B}$, and challenges $X_{0}, Y_{0}$ with respect to public keys $A, B$. Otherwise $\mathcal{S}$ aborts.

\footnotetext{
${ }^{3}$ To simulate consistently the ephemeral exponent leakage on $\hat{B}, \mathcal{S}$ has to assign values to $\bar{H}$ query with a partially unknown input, namely the incoming ephemeral key is unknown. For these queries, random values are taken in $\{0,1\}^{l}$ as $\bar{H}\left(Y, \star, \hat{B}, \hat{P}_{l}\right)$ and $\bar{H}\left(\star, Y, \hat{B}, \hat{P}_{l}\right)$; and when $\mathcal{S}$ is later queried $\bar{H}\left(Y, X, \hat{B}, \hat{P}_{l}\right)$ (resp. $\bar{H}\left(X, Y, \hat{B}, \hat{P}_{l}\right)$, it responds with $\bar{H}\left(Y, \star, \hat{B}, \hat{P}_{l}\right)$ (resp. $\left.\bar{H}\left(\star, Y, \hat{B}, \hat{P}_{l}\right)\right)$.
} 
The simulation of $\mathcal{A}$ 's environment is perfect, except with negligible probability. The deviation happens when the same $Y$ is chosen twice as ephemeral key in two sessions at $\hat{B}$ with the same peer $\hat{P}_{l}$; this happens with probability less than $m / q$, which is negligible. Hence, under this simulation, $\mathcal{A}$ succeeds with non-negligible probability in E.2. And when $\mathcal{A}$ outputs a correct forgery, and $\mathcal{S}$ guesses correctly the test session, $\mathcal{S}$ outputs a valid FDCR forgery on messages $\hat{A}$, $\hat{B}$, and challenges $X_{0}, Y_{0}$, with respect to the public keys $A$ and $B$; contradicting Proposition 5 .

Neither E.1 nor E.2, can happen with non-negligible probability; the FHMQV protocol is ck-secure.

\subsubsection{Resistance to Reflection Attacks}

We show here that the FHMQV protocol provides resistance to reflection attacks (for ck-fresh sessions). A session with identifier sid $=(\hat{A}, \hat{A}, X, Y)$ is said to be ck-fresh if none of these conditions hold:

- $\mathcal{A}$ issues a SecretExponentReveal, a SecretGroupElementReveal, or a SessionKeyReveal query on sid or sid* (if sid* exists);

- sid ${ }^{*}$ does not exist and $\mathcal{A}$ makes a StaticKeyReveal query on $\hat{A}$.

Recall that under the RO model, guessing and key replication attacks cannot succeed, except with negligible probability. It suffices to show that no polynomially bounded adversary can compute, with non-negligible success probability, the session signature of a ck-fresh session $\left(\hat{A}, \hat{A}, X_{0}, Y_{0}\right)$.

Let $F$ be the event " $\mathcal{A}$ succeeds in forging the session signature of some ck-fresh session $\left(\hat{A}, \hat{A}, X_{0}, Y_{0}\right)$." The event $F$ divides in F.1: "A succeeds in forging the session signature of some ck-fresh session $\left(\hat{A}, \hat{A}, X_{0}, Y_{0}\right)$, which matching session exists", and F.2: "A succeeds in forging the session signature of some ck-fresh session $\left(\hat{A}, \hat{A}, X_{0}, Y_{0}\right)$ without matching session." It thus suffices to show that neither F.1 nor F.2 can happen with non-negligible probability.

Analysis of F.1. If F.1 happens with non-negligible probability, using $\mathcal{A}$, we build a polynomial time $\mathrm{CDH}$ solver which succeeds with non-negligible probability. To do this, we reuse the simulation in the analysis of E.1, except the following differences.

- $\mathcal{S}$ takes as input $a, X_{0}, Y_{0} ; a \in_{R}[1, q-1], X_{0}, Y_{0} \in_{R} \mathcal{G}^{*}$.

- $\hat{B}$ 's key pair and identity are set to that of $\hat{A}$.

$\mathcal{A}$ 's simulated environment remains perfect except with negligible probability; and if $\mathcal{A}$ succeeds with non-negligible probability, in event F.1, then under this simulation it outputs with nonnegligible probability a valid signature forgery $\sigma_{0}$. And then $\mathcal{S}$ outputs with non-negligible probability $C D H\left(X_{0}, Y_{0}\right)$ from $\sigma_{0}, a, d$, and $e$. This shows that under the $\mathrm{CDH}$ assumption and RO model, F.1 cannot happen, except with negligible probability.

Analysis of $\boldsymbol{F . 2 .}$ If $F .2$ occurs with non-negligible probability, using $\mathcal{A}$, we build a polynomial time machine, which given $A=G^{a}$ outputs with non-negligible probability $G^{\left(a^{2}\right)}$. Such a "squaring" CDH solver can in turn be used as a general CHD solver, which succeeds with non-negligible probability [16].

We simulate $\mathcal{A}$ 's environment as in the analysis of E.1, with the following modifications.

- $\mathcal{S}$ takes as input $A \in_{R} \mathcal{G}^{*}(\hat{A}=\hat{B})$.

- $\hat{A}$ 's public key is set to $A$; its roles are simulated in the same way as that of $\hat{B}$ in the analysis E.2.

- When $\mathcal{A}$ activates the $t$-th session at $\hat{A}, \mathcal{S}$ chooses $x_{0} \in_{R}[1, q-1]$, and provides $\mathcal{A}$ with the message $\left(\hat{A}, \hat{A}, X_{0}=G^{x_{0}}\right)$; and if $\mathcal{A}$ activates at $\hat{B}$ a session matching $t$-th session at $\hat{A}, \mathcal{S}$ aborts.

- If $\mathcal{A}$ issues a static key reveal on $\hat{A}, \mathcal{S}$ aborts. 
The simulated environment remains perfect, except with negligible probability; and if $\mathcal{A}$ succeeds with non-negligible probability in F.2, then under this simulation, it outputs with nonnegligible probability a valid forgery $\sigma_{0}$ of the test session's signature. And then since $\mathcal{S}$ knows $x_{0}$ it outputs with non-negligible probability

$$
\left(\left(\sigma_{0}\right)\left(Y_{0} A^{e}\right)^{-x_{0}}\right)^{d^{-1}}=\left(\left(Y_{0} A^{e}\right)^{x_{0}+d a}\left(Y_{0} A^{e}\right)^{-x_{0}}\right)^{d^{-1}}=\left(\left(Y_{0} A^{e}\right)^{d a}\right)^{d^{-1}}=\left(Y_{0} A^{e}\right)^{a}=A^{y_{0}+e a} .
$$

Hence, given a public key $A, \mathcal{S}$ outputs with non-negligible probability a valid and fresh FXCR signature, on message $(\hat{A}, \hat{B})$ (concatenation of $\hat{A}$ and $\hat{B}$ ), and challenge $A$ (the challenge equals the public key) with respect to the public key $A$. Using the "oracle replay" technique (as in the proof of Proposition 4), $\mathcal{S}$ yields a polynomial machine, which given $A=G^{a}$, outputs with non-negligible probability $A^{a}=G^{\left(a^{2}\right)}$; contradicting the CDH assumption.

\subsection{Ephemeral Private Keys Leakage Resilience (eck-security)}

The arguments for this security attribute do not derive from the analysis in section 4 . The reason is that we cannot simultaneously and consistently simulate both SecretExponentReveal and EphemeralKeyReveal.

\subsubsection{Hashed Full Dual Challenge Response Scheme}

Definition 10 (Hashed FDCR (HFDCR) signature scheme). Let $\hat{A}, \hat{B}$ be two entities with public keys $A, B \in_{R} \mathcal{G}^{*}$. The HFDCR signature of $\hat{A}$ and $\hat{B}$ on messages $m_{1}, m_{2}$ is

$$
\operatorname{HFDCR}_{A, B}\left(X, Y, m_{1}, m_{2}\right)=H\left(\sigma, m_{1}, m_{2}, X, Y\right),
$$

where $\sigma$ is the FDCR signature of $\hat{A}$ and $\hat{B}$ on messages $m_{1}, m_{2}$, and challenges $X, Y$.

Definition 11 (Security of the HFDCR Signature Scheme). Let $\hat{A}, \hat{B}$ be two entities with public keys $A, B \in_{R} \mathcal{G}^{*}$. The HFDCR scheme is secure, if given $A, B$, and $x_{0}, y_{0} \in_{R}[1, q-$ 1] together with hashing and signing oracles, no adaptive probabilistic polynomial time attacker can produce with non negligible success probability a triple $\left(m_{1_{0}}, m_{2_{0}}, \pi_{0}\right)$ such that: $H F D C R_{A, B}\left(X_{0}, Y_{0}, m_{1_{0}}, m_{2_{0}}\right)=\pi_{0}$, and $\pi_{0}$ was not obtained from the signing oracle with a query on a quadruple $\left(X_{0}, Y_{0}, m_{1}^{\prime}, m_{2}^{\prime}\right)$ such that $\left(m_{1_{0}}, m_{2_{0}}\right)=\left(m_{1}^{\prime}, m_{2}^{\prime}\right)$.

For the HFDRC security arguments, we need the Gap Diffie-Hellman (GDH) assumption.

Definition $12([21])$. Let $\mathcal{G}=\langle G\rangle$ be a cyclic group. An algorithm is said to be a Decisional Diffie-Hellman Oracle (DDHO) for $\mathcal{G}$, if on input $G, X=G^{x}, Y=G^{y}, Z \in \mathcal{G}$, it outputs 1 if and only if $Z=G^{x y}$. The Gap Diffie-Hellman (GDH) assumption is said to hold in $\mathcal{G}$, if given a DDHO for $\mathcal{G}$, no polynomially bounded algorithm can solve the $\mathrm{CDH}$ problem in $\mathcal{G}$, with non-negligible success probability.

We now prove the security of the HFDCR scheme, under the GDH assumption and the RO model.

Proposition 7. Under the $G D H$ assumption in $\mathcal{G}$, and the $R O$ model, the HFDCR scheme is secure in the sense of definition 11.

Proof. Suppose a polynomially bounded attacker $\mathcal{A}$, which given a DDHO, $A, B \in_{R} \mathcal{G}^{*}$, and $x_{0}, y_{0} \in_{R}[1, q-1]$, outputs with non-negligible success probability a valid and fresh $\operatorname{HFDCR}_{A, B}$ signature on some messages $m_{1_{0}}, m_{2_{0}}$ with respect to challenges $X_{0}=G^{x_{0}}, Y=G^{y_{0}}$.

Non-matching HFDCR signature queries cannot have the same signature value, except with negligible probability. And guessing the output of the hash function cannot be performed with non-negligible success probability. We can thus suppose that $\mathcal{A}$ succeeds with non-negligible probability in forging attack. Using $\mathcal{A}$ and a DDHO, we build a polynomial time $\mathrm{CDH}$ solver $\mathcal{S}$ which succeeds with non-negligible probability. The solver $\mathcal{S}$ provides $\mathcal{A}$ with random coins and simulates the signature queries; it takes as input $A, B \in_{R} \mathcal{G}^{*}, x_{0}, y_{0} \in_{R}[1, q-1]$, and outputs with non-negligible probability $C D H(A, B)$. 
(1) At $\mathcal{A}$ 's $\bar{H}$ digest query on $\left(X, Y, m_{1}, m_{2}\right), \mathcal{S}$ does the following:

- If a value is already assigned to $\bar{H}\left(X, Y, m_{1}, m_{2}\right), \mathcal{S}$ provides $\mathcal{A}$ with the value of $\bar{H}\left(X, Y, m_{1}, m_{2}\right)$.

- Else $\mathcal{S}$ chooses $d \in_{R}\{0,1\}^{l}$, sets $\bar{H}\left(X, Y, m_{1}, m_{2}\right)=d$, and provides $\mathcal{A}$ with $d$.

(2) At $\mathcal{A}$ 's signature query on $\left(X, Y, m_{1}, m_{2}\right), \mathcal{S}$ responds as follows:

- If a value is already assigned to $H F D C R_{A, B}\left(X, Y, m_{1}, m_{2}\right)$, then $\mathcal{S}$ returns the value of $H F D C R_{A, B}\left(X, Y, m_{1}, m_{2}\right)$; else $\mathcal{S}$ takes $\pi \in_{R}\{0,1\}^{\lambda}$, sets $H F D C R_{A, B}\left(X, Y, m_{1}, m_{2}\right)$ $=\pi$, and provides $\mathcal{A}$ with $\pi$.

- If no value is assigned to $\bar{H}\left(X, Y, m_{1}, m_{2}\right)$ (resp. $\left.\bar{H}\left(Y, X, m_{1}, m_{2}\right)\right), \mathcal{S}$ chooses $d \in_{R}$ $\{0,1\}^{l}$ and sets $\bar{H}\left(X, Y, m_{1}, m_{2}\right)=d\left(\right.$ resp. $\left.\bar{H}\left(Y, X, m_{1}, m_{2}\right)=d\right)$.

(3) At $\mathcal{A}$ 's digest query on $\left(\sigma, m_{1}, m_{2}, X, Y\right), \mathcal{S}$ does the following:

- If a value is assigned to $H F D C R_{A, B}\left(X, Y, m_{1}, m_{2}\right)$, and if $\sigma=C D H\left(X A^{d}, Y B^{e}\right)$, where $d=\bar{H}\left(X, Y, m_{1}, m_{2}\right), e=\bar{H}\left(Y, X, m_{1}, m_{2}\right)$ (if a value is already assigned to $H F D C R_{A, B}\left(X, Y, m_{1}, m_{2}\right), d$ and $e$ are defined, and the verification is performed using the DDHO), $\mathcal{S}$ returns the value of $\operatorname{HFDCR}_{A, B}\left(X, Y, m_{1}, m_{2}\right)$.

- Else, $(i)$ if the same query was made previously, $\mathcal{S}$ returns the previously returned value; (ii) else $\mathcal{S}$ chooses $\pi \in_{R}\{0,1\}^{\lambda}$, sets $H\left(\sigma, m_{1}, m_{2}, X, Y\right)=\pi$, and provides $\mathcal{A}$ with $\pi$.

- If no value is assigned to $\bar{H}\left(X, Y, m_{1}, m_{2}\right)$ (resp. $\left.\bar{H}\left(Y, X, m_{1}, m_{2}\right)\right), \mathcal{S}$ chooses $d \in_{R}$ $\{0,1\}^{l}$ and sets $\bar{H}\left(X, Y, m_{1}, m_{2}\right)=d$ (resp. $\left.\bar{H}\left(Y, X, m_{1}, m_{2}\right)=d\right) ;$ and if $\sigma=$ $C D H\left(X A^{d}, Y B^{e}\right), \mathcal{S}$ sets $H F D C R_{A, B}\left(X, Y, m_{1}, m_{2}\right)=\pi$.

(4) If $\mathcal{A}$ halts with a forgery $\pi_{0}, X_{0}, Y_{0}, m_{1_{0}}, m_{2_{0}}, \mathcal{S}$ verifies that the digests value $\pi_{0}$ was queried from the random oracle, as $H\left(\sigma_{0}, m_{1_{0}}, m_{2_{0}}, X_{0}, Y_{0}\right)$ for some $\sigma_{0}$, and that $\sigma_{0}=C D H\left(X_{0} A^{d}\right.$, $\left.Y_{0} B^{e}\right)$, where $d=\bar{H}\left(X_{0}, Y_{0}, m_{1_{0}}, m_{2_{0}}\right)$, and $e=\bar{H}\left(Y_{0}, X_{0}, m_{1_{0}}, m_{2_{0}}\right)$ (if $\pi_{0}$ was queried from the hashing oracle, $\bar{H}\left(X_{0}, Y_{0}, m_{1_{0}}, m_{2_{0}}\right)$ and $\bar{H}\left(Y_{0}, X_{0}, m_{1_{0}}, m_{2_{0}}\right)$ are defined).

Under the RO model, $\mathcal{A}$ 's simulated environment is perfect except with negligible probability; hence if $\mathcal{A}$ succeeds with non-negligible probability in forging a HFDRC signature, it succeeds under this simulation with the same probability, except a negligible difference. Since $\mathcal{S}$ knows $x_{0}, y_{0}$, and $\mathcal{A}$ succeeds with non-negligible probability, $\mathcal{S}$ outputs with non-negligible probability

$$
\begin{aligned}
\left(\left(\left(\sigma_{0}\right)\left(Y_{0} B^{e}\right)^{-x_{0}}\right)^{d^{-1}} A^{-y_{0}}\right)^{e^{-1}} & =\left(\left(\left(Y_{0} B^{e}\right)^{x_{0}+d a}\left(Y_{0} B^{e}\right)^{-x_{0}}\right)^{d^{-1}} A^{-y_{0}}\right)^{e^{-1}} \\
& =\left(\left(\left(Y_{0} B^{e}\right)^{d a}\right)^{d^{-1}} A^{-y_{0}}\right)^{e^{-1}}=\left(\left(Y_{0} B^{e}\right)^{a} A^{-y_{0}}\right)^{e^{-1}} \\
& =\left(A^{y_{0}+e b} A^{-y_{0}}\right)^{e^{-1}}=C D H(A, B) ;
\end{aligned}
$$

contradicting the GDH assumption.

\subsubsection{Application to FHMQV}

Using the HFDCR security, we now show that the FHMQV protocol meets the eck-security definition.

Proposition 8. Under the $G D H$ assumption in $\mathcal{G}$, and the $R O$ model, the FHMQV protocol is eck-secure.

Proof of Proposition 8. Since guessing and key replication attacks cannot succeed with nonnegligible probability, suppose $\mathcal{A}$ succeeds with non-negligible probability in forging attack.

Let $E$ ' be the event ' $\mathcal{A}$ succeeds in forging an eck-fresh session signature." $E$ ' divides in $E$.1: " $\mathcal{A}$ succeeds in forging the session signature of some eck-fresh session, which matching session exists", and E'2: "A succeeds in forging the session signature of some eck-fresh session, without matching session." It suffices to show that neither E'.1 nor E'2 can happen with non-negligible probability. 
Analysis of E.1. Since the test session sid is required to be eck-fresh and sid* exists, the strongest queries that $\mathcal{A}$ can perform are:(i) StaticKeyReveal queries on both $\hat{A}$ and $\hat{B}$; (ii) EphemeralKeyReveal queries on both sid and sid*; (iii) a StaticKeyReveal query on $\hat{A}$ and an EphemeralKeyReveal query on sid*; (iv) an EphemeralKeyReveal query on sid and a StaticKeyReveal query on $\hat{B}$. As from any polynomial time machine which succeeds in E:.1, and performs weaker queries than those above, one can build a polynomial time machine, which succeeds with same probability and performs one of the strongest queries, it suffices to show that none of the events

E'1.1: "E'.1 $\wedge \mathcal{A}$ performs StaticKeyReveal queries on both $\hat{A}$ and $\hat{B}$ ",

E'1.2: "E'1 $\wedge \mathcal{A}$ performs EphemeralKeyReveal queries on both sid and sid"",

E.1.3: "E.1 $\wedge \mathcal{A}$ performs a StaticKeyReveal query on $\hat{A}$ and an EphemeralKeyReveal query on sid", E.1.4: "E'.1 $\wedge \mathcal{A}$ performs an EphemeralKeyReveal query on sid and a StaticKeyReveal query on $\hat{B}$ ", can occur with non-negligible probability.

Analysis of E'1.1. Suppose that E'1.1 occurs with non-negligible probability; using $\mathcal{A}$, we build a polynomial time $\mathrm{CDH}$ solver which succeeds with non-negligible probability. For this purpose, we use the same simulation as in the analysis of E.1 with the following modifications (recall that the allowed queries are that from Set 2):

- If $\mathcal{A}$ issues a SessionKeyReveal, EphemeralKeyReveal, or an StaticKeyReveal query, $\mathcal{S}$ answers faithfully.

- In any of the following situations, $\mathcal{S}$ aborts.

- $\mathcal{A}$ halts with a test session different from the $t$-th session at $\hat{A}$.

- $\mathcal{A}$ issues a SessionKeyReveal query on the $t$-th session at $\hat{A}$ or its matching session.

- $\mathcal{A}$ issues an EphemeralKeyReveal query on the $t$-th session at $\hat{A}$ or its matching session.

- $\mathcal{A}$ issues an EstablishParty query on $\hat{A}$ or $\hat{B}$.

The simulated environment remains perfect, except with negligible probability. The probability of guessing correctly the test session is $\left(n^{2} m\right)^{-1}$. If $\mathcal{A}$ succeeds with non-negligible probability in E'.1.1, under this simulation $\mathcal{A}$ outputs a valid forgery of the $t$-th session at $\hat{A}$ with non-negligible probability. Hence $\mathcal{S}$ outputs $C D H\left(X_{0}, Y_{0}\right)$ (from $\mathcal{A}$ 's forgery and $a, b, d, e$ ) with non-negligible probability.

Analysis of E'1.2. We reuse the simulation of the analysis of E.1, with the following modifications:

- $\mathcal{S}$ takes as input $x_{0}, y_{0} \in_{R}[1, q-1]$, and $A, B \in_{R} \mathcal{G}^{*}$.

- $\hat{A}$ and $\hat{B}$ 's public keys are set to $A$ and $B$ (the private keys are unknown).

- At $\mathcal{A}$ 's $\operatorname{Send}\left(\hat{P}_{m}, \hat{P}_{l}, Y\right)$ query, with $\hat{P}_{l}=\hat{A}$ or $\hat{B}, \mathcal{S}$ responds as follows.

- $\mathcal{S}$ chooses $x \in_{R}[1, q-1]$, computes $X=G^{x}$, creates a session state with identifier $\left(\hat{P}_{l}, \hat{P}_{m}, X, Y\right)$, provides $\mathcal{A}$ with the outgoing message $\left(\hat{P}_{l}, \hat{P}_{m}, X\right)$.

$-\mathcal{S}$ chooses $\pi \in_{R}\{0,1\}^{\lambda}, d, e \in_{R}\{0,1\}^{l}$ and sets $\operatorname{HFDCR}_{P_{m}, P_{l}}\left(Y, X, \hat{P}_{m}, \hat{P}_{l}\right)=\pi$, $\bar{H}\left(Y, X, \hat{P}_{m}, \hat{P}_{l}\right)=d$, and $\bar{H}\left(X, Y, \hat{P}_{m}, \hat{P}_{l}\right)=e$.

- At $\operatorname{Send}\left(\hat{P}_{l}, \hat{P}_{m}, X, Y\right)$ query, with $\hat{P}_{l}=\hat{A}$ or $\hat{B}, \mathcal{S}$ answers as follows:

- $\mathcal{S}$ updates the session identifier $\left(\hat{P}_{l}, \hat{P}_{m}, X, \star\right)$ (if any) to $\left(\hat{P}_{l}, \hat{P}_{m}, X, Y\right)$.

- If a no value is assigned to $H F D C R_{P_{l}, P_{m}}\left(X, Y, \hat{P}_{l}, \hat{P}_{m}\right), \mathcal{S}$ chooses $\pi \in_{R}\{0,1\}^{\lambda}$ and sets $H F D C R_{P_{l}, P_{m}}\left(X, Y, \hat{P}_{l}, \hat{P}_{m}\right)=\pi$; if no value is assigned to $\bar{H}\left(X, Y, \hat{P}_{l}, \hat{P}_{m}\right)$ (resp. $\left.\bar{H}\left(Y, X, \hat{P}_{l}, \hat{P}_{m}\right)\right), \mathcal{S}$ chooses $d \in_{R}\{0,1\}^{l}$ and sets $\bar{H}\left(X, Y, \hat{P}_{l}, \hat{P}_{m}\right)=d$ (resp. $\left.\bar{H}\left(Y, X, \hat{P}_{l}, \hat{P}_{m}\right)=d\right)$.

- At $\mathcal{A}$ 's digest query on $\left(\sigma, \hat{P}_{l}, \hat{P}_{m}, X, Y\right)$, with $\hat{P}_{l}=\hat{A}$ or $\hat{B}$, or $\hat{P}_{m}=\hat{A}$ or $\hat{B}, \mathcal{S}$ does the following:

- If a value is already assigned to $H F D C R_{P_{l}, P_{m}}\left(X, Y, \hat{P}_{l}, \hat{P}_{m}\right)$, and if $\sigma=C D H\left(X P_{l}^{d}\right.$, $\left.Y P_{m}^{e}\right)$, where $d=\bar{H}\left(X, Y, \hat{P}_{l}, \hat{P}_{m}\right), e=\bar{H}\left(Y, X, \hat{P}_{l}, \hat{P}_{m}\right)\left(\right.$ if $H F D C R_{P_{l}, P_{m}}\left(X, Y, \hat{P}_{l}, \hat{P}_{m}\right)$ is already defined, the values of $d$ and $e$ are already assigned), $\mathcal{S}$ returns the value of $H F D C R_{P_{l}, P_{m}}\left(X, Y, \hat{P}_{l}, \hat{P}_{m}\right)$. 
- Else, $\mathcal{S}$ chooses $\pi \in_{R}\{0,1\}^{\lambda}$, sets $H\left(\sigma, \hat{P}_{l}, \hat{P}_{m}, X, Y\right)=\pi$, and provides $\mathcal{A}$ with $\pi$.

- If no value is assigned to $\bar{H}\left(X, Y, \hat{P}_{l}, \hat{P}_{m}\right)$ (resp. $\left.\bar{H}\left(Y, X, \hat{P}_{l}, \hat{P}_{m}\right)\right), \mathcal{S}$ chooses $d \in_{R}$ $\{0,1\}^{\lambda}$ and sets $\bar{H}\left(X, Y, \hat{P}_{l}, \hat{P}_{m}\right)\left(\operatorname{resp} . \bar{H}\left(Y, X, \hat{P}_{l}, \hat{P}_{m}\right)\right)=d$; and if $\sigma=C D H\left(X P_{l}^{d}\right.$, $\left.Y P_{m}^{e}\right)$ (this is verified using the DDHO), $\mathcal{S}$ sets $\operatorname{HFDCR}_{P_{l}, P_{m}}\left(X, Y, \hat{P}_{l}, \hat{P}_{m}\right)=\pi$.

- When $\mathcal{A}$ activates the $t$-th session at $\hat{A}$, if the peer is not $\hat{B}, \mathcal{S}$ aborts; otherwise $\mathcal{S}$ provides $\mathcal{A}$ with the outgoing message $\left(\hat{A}, \hat{B}, X_{0}=G^{x_{0}}\right)$.

- When $\mathcal{A}$ activates the session matching $t$-th session at $\hat{A}, \mathcal{S}$ provides $\mathcal{A}$ with $\left(\hat{B}, \hat{A}, Y_{0}=\right.$ $\left.G^{y_{0}}\right)$.

- If $\mathcal{A}$ issues an EphemeralKeyReveal query on the $t$-th session at $\hat{A}$ or its matching session, $\mathcal{S}$ answers faithfully.

- In the following situations $\mathcal{S}$ aborts.

$-\mathcal{A}$ halts with a test session different from the $t$-th session at $\hat{A}$.

- $\mathcal{A}$ issues a StaticKeyReveal query on $\hat{A}$ or $\hat{B}$.

- $\mathcal{A}$ issues an EstablishParty query on $\hat{A}$ or $\hat{B}$.

The simulated environment remains perfect, except with negligible probability, and the probability of guessing correctly the test session is $\left(n^{2} m\right)^{-1}$. If $\mathcal{A}$ succeeds with a FDCR signature $\sigma_{0}$, and $\mathcal{S}$ guesses correctly the test session, $\mathcal{S}$ outputs a valid HFDCR forgery $\pi_{0}=H\left(\sigma_{0}, \hat{A}, \hat{B}, X_{0}, Y_{0}\right)$, contradicting Proposition 7.

Analysis of E.1.3 and E.1.4. Since $\hat{A}$ and $\hat{B}$ roles are symmetrical in E'.1.3 and E.1.4, it suffices to show that E'.1.3 cannot occur with non-negligible probability. We modify, the simulation used in the analysis of $E .1$ as follows:

- $\mathcal{S}$ takes as input $X_{0}, B \in_{R} \mathcal{G}^{*}$.

- $\hat{B}$ 's public key is set to $B$ (the private key is unknown to $\mathcal{S}$ ), and $\hat{A}$ 's key pair is ( $a=$ $\left.p_{i}, G^{a}\right), p_{i} \in_{R}[1, q-1]$.

- At $\mathcal{A}$ 's $\operatorname{Send}\left(\hat{P}_{m}, \hat{B}, X\right)$ query, $\mathcal{S}$ responds as follows:

- $\mathcal{S}$ chooses $y \in_{R}[1, q-1]$, computes $Y=G^{y}$, creates a session state with identifier $\left(\hat{B}, \hat{P}_{m}, Y, X\right)$, and provides $\mathcal{A}$ with the outgoing message $\left(\hat{B}, \hat{P}_{m}, Y\right)$.

$-\mathcal{S}$ chooses $\pi \in_{R}\{0,1\}^{\lambda}, d, e \in_{R}\{0,1\}^{l}$, and sets $H_{F D C R_{P_{m}, B}}\left(X, Y, \hat{P}_{m}, \hat{B}\right)=\pi$, $\bar{H}\left(X, Y, \hat{P}_{m}, \hat{B}\right)=d$, and $\bar{H}\left(Y, X, \hat{P}_{m}, \hat{B}\right)=e$.

- At $\operatorname{Send}\left(\hat{B}, \hat{P}_{m}, Y, X\right)$ query:

- $\mathcal{S}$ updates the session identifier $\left(\hat{B}, \hat{P}_{m}, Y, \star\right)$ to $\left(\hat{B}, \hat{P}_{m}, Y, X\right)$.

- If no value is assigned to $\operatorname{HFDCR}_{B, P_{m}}\left(Y, X, \hat{B}, \hat{P}_{m}\right), \mathcal{S}$ chooses $\pi \in_{R}\{0,1\}^{\lambda}$ and sets $H F D C R_{B, P_{m}}\left(Y, X, \hat{B}, \hat{P}_{m}\right)=\pi$; and if no value is assigned to $\bar{H}\left(Y, X, \hat{B}, \hat{P}_{m}\right)$ (resp. $\left.\bar{H}\left(X, Y, \hat{B}, \hat{P}_{m}\right)\right), \mathcal{S}$ chooses $d \in_{R}\{0,1\}^{l}$ and sets $\bar{H}\left(Y, X, \hat{B}, \hat{P}_{m}\right)=d$ (resp. $\left.\bar{H}\left(X, Y, \hat{B}, \hat{P}_{m}\right)=d\right)$.

- At $\mathcal{A}$ 's digest query on $\left(\sigma, \hat{P}_{l}, \hat{P}_{m}, X, Y\right)$, with $\hat{P}_{l}=\hat{B}$, or $\hat{P}_{m}=\hat{B}, \mathcal{S}$ responds as follows:

- If a value is assigned to $H F D C R_{P_{l}, P_{m}}\left(X, Y, \hat{P}_{l}, \hat{P}_{m}\right)$, and if $\sigma=C D H\left(X P_{l}^{d}, Y P_{m}^{e}\right)$, where $d=\bar{H}\left(X, Y, \hat{P}_{l}, \hat{P}_{m}\right), e=\bar{H}\left(Y, X, \hat{P}_{l}, \hat{P}_{m}\right), \mathcal{S}$ returns $\operatorname{HFDCR}_{P_{l}, P_{m}}\left(X, Y, \hat{P}_{l}\right.$, $\left.\hat{P}_{m}\right)$;

- Else, $\mathcal{S}$ chooses $\pi \in_{R}\{0,1\}^{\lambda}$, sets $H\left(\sigma, \hat{P}_{l}, \hat{P}_{m}, X, Y\right)=\pi$, and provides $\mathcal{A}$ with $\pi$;

- If no value is assigned to $\bar{H}\left(X, Y, \hat{P}_{l}, \hat{P}_{m}\right)$ (resp. $\left.\bar{H}\left(Y, X, \hat{P}_{l}, \hat{P}_{m}\right)\right), \mathcal{S}$ chooses $d \in_{R}$ $\{0,1\}^{\lambda}$ and sets $\bar{H}\left(X, Y, \hat{P}_{l}, \hat{P}_{m}\right)=d$ (resp. $\left.\bar{H}\left(Y, X, \hat{P}_{l}, \hat{P}_{m}\right)=d\right)$; and if $\sigma=$ $C D H\left(X P_{l}^{d}, Y P_{m}^{e}\right), \mathcal{S}$ sets $H F D C R_{P_{l}, P_{m}}\left(X, Y, \hat{P}_{l}, \hat{P}_{m}\right)=\pi$.

- When $\mathcal{A}$ activates the $t$-th session at $\hat{A}$, if the peer is not $\hat{B}, \mathcal{S}$ aborts; otherwise, $\mathcal{S}$ provides $\mathcal{A}$ with the outgoing message $\left(\hat{A}, \hat{B}, X_{0}\right)$.

- When $\mathcal{A}$ activates the session matching $t$-th session at $\hat{A}, \mathcal{S}$ chooses $y_{0} \in_{R}[1, q-1]$, and provides $\mathcal{A}$ with $\left(\hat{B}, \hat{A}, Y_{0}=G^{y_{0}}\right)$.

- If $\mathcal{A}$ issues an EphemeralKeyReveal query on the session matching the $t$-th session at $\hat{A}$, $\mathcal{S}$ answers faithfully.

- In any of the following situations $\mathcal{S}$ aborts.

$-\mathcal{A}$ halts with a test session different from the $t$-th session at $\hat{A}$.

- $\mathcal{A}$ issues a StaticKeyReveal query on $\hat{B}$. 
- $\mathcal{A}$ issues an EphemeralKeyReveal query on the $t$-th session at $\hat{A}$.

- $\mathcal{A}$ issues an EstablishParty query on $\hat{A}$ or $\hat{B}$.

The simulation remains perfect, except with negligible probability; if $\mathcal{A}$ succeeds with nonnegligible probability in event $E$ :.1.3, $\mathcal{S}$ outputs with non-negligible probability $C D H\left(X_{0}, B\right)$, from $\mathcal{A}$ 's forgery (and $a, y_{0}, d$, and $e$ ); contradicting the GDH assumption.

Under the GDH assumption and the RO model, none of the events E'1.1, E'1.2, E'.1.3, or E'1.4 occur with non-negligible probability, this shows that E'.1 cannot happen, except with negligible probability.

Analysis of $\boldsymbol{E}^{\prime} \cdot 2$ (sketch). The strongest query that $\mathcal{A}$ can perform in E'2 are: a StaticKeyReveal query on $\hat{A}$ or an EphemeralKeyReveal query on sid (but not both). It thus suffices to show that neither E'2.1: "E'2 $\wedge \mathcal{A}$ performs a StaticKeyReveal query on $\hat{A}$ " nor $E$ '.2.2: "E'.2 $\wedge \mathcal{A}$ performs an EphemeralKeyReveal query on sid" can occur with non-negligible probability.

To show that E'2.1 cannot happen with non-negligible probability, the simulation used in the analysis of E'.1.3 can be modified such that if $\mathcal{A}$ activates a session matching the $t$-th session at $\hat{A}, \mathcal{S}$ aborts. Since $\mathcal{A}$ succeeds with non-negligible probability, using $\mathcal{A}, \mathcal{S}$ outputs with nonnegligible probability $\left(Y_{0} B^{e}\right)^{x_{0}}$ from $\mathcal{A}$ forgery and $a$. Hence, from the forking lemma, using $\mathcal{S}$, one can build a polynomial machine $\mathcal{S}^{\prime}$ which given $X_{0}, B$, outputs with non-negligible probability $C D H\left(X_{0}, B\right)$. One can then show that under the RO model and the GDH assumption, E'2.1 cannot occur, except with negligible probability.

For the analysis of E'2.2, the simulation the analysis of E'.1.2 can be modified to take as input $x_{0}, A, B$, and aborts when $\mathcal{A}$ activates a session matching the $t$-th session at $\hat{A}$. If $\mathcal{A}$ succeeds in E'.2.2 (and then under this simulation), using $\mathcal{A}$ 's forgery, $\mathcal{S}$ outputs $\left(Y_{0} B^{e}\right)^{a}$; and using the "oracle replay" technique, $\mathcal{S}$ can be transformed into a machine which given $A, B$ outputs with non-negligible probability $C D H(A, B)$, contradicting the GDH assumption.

Reflection Attacks Resilience (sketch). With arguments similar to that of subsection 5.1.3, one can show that reflection attacks cannot hold against eck-fresh sessions. Indeed, if $\hat{A}=\hat{B}$, and if the session matching the test session exists, $\mathcal{A}$ is not allowed to perform both a StaticKeyReveal query on $\hat{A}$, and an EphemeralKeyReveal query on the test session or its matching session. And if the test session's matching session does not exist, a StaticKeyReveal query on $\hat{A}$ is not allowed.

The analysis of E'.1.1 remains valid if $\hat{A}=\hat{B}$. And in $E^{\prime} .1 .2$, when $\hat{A}=\hat{B}$ a polynomial time successful attacker, yields a polynomial machine which given $A=G^{a}$ outputs $G^{\left(a^{2}\right)}$. Under these restrictions on the allowed queries if $\hat{A}=\hat{B}$, and with minor modifications in the analysis of E'1.1 and E'1.2, one can show that under the RO model and the GDH assumption, the FHMQV protocol provides resistance to reflection attacks for eck-fresh sessions.

\subsection{Main Differences between FHMQV and HMQV Security Arguments}

We summarize the most important differences between the HMQV and FHMQV security arguments.

Building blocks and adversary model. The design of FHMQV relies on the FXCR and FDCR signature schemes. While in the XCR scheme as in the FXCR scheme, both $s_{A}$ and $x$ leakages in the same session imply $\hat{A}$ 's private key discloser. In the FXCR scheme, an adversary which learns $s_{A}$ is unable to forge $A$ 's signature. The FHMQV adversary model allows ephemeral secret exponent leakage. The impersonation and man in the middle attacks we presented in section 2 do not hold against FHMQV.

Key replication attacks resilience. At session key derivation in FHMQV, ephemeral keys and peers identities are hashed with the session's FDCR signature $(K=H(\sigma, \hat{A}, \hat{B}, X, Y))$. Since non matching sessions cannot have (except with negligible probability) the same ephemeral keys, and non matching digest queries cannot have (except with negligible 
probability) the same digest value, the analysis of key replication attacks is immediate for the FHMQV protocol.

Ephemeral private keys leakage resilience. To show this security attribute for FHMQV, we define the Hashed FDCR signature scheme. For the HMQV protocol, it is used a hashed variant of the XCR, namely the HCR signature scheme [11]. While both HFDCR and HCR security arguments rely on the GDH assumption, for the HFDCR scheme the Knowledge of Exponent Assumption (KEA1) [4] is not needed, whereas it is required for the HCR scheme.

\section{The FHMQV-C Protocol}

As shown in [11], no implictely authenticated two message key agreement protocol can meet the perfect forward secrecy security attribute; key confirmation security attribute (for both peers) cannot be achieved also. Nevertheless these security attributes may be desirable; the FHMQV protocol can be added with a third message, yielding the FHMQV-C protocol, we describe in Protocol 6; $\mathrm{KDF}_{1}$ and $\mathrm{KDF}_{2}$ are key derivation functions, and $\mathrm{MAC}$ a message authentication code. If any verification fails, the execution aborts.

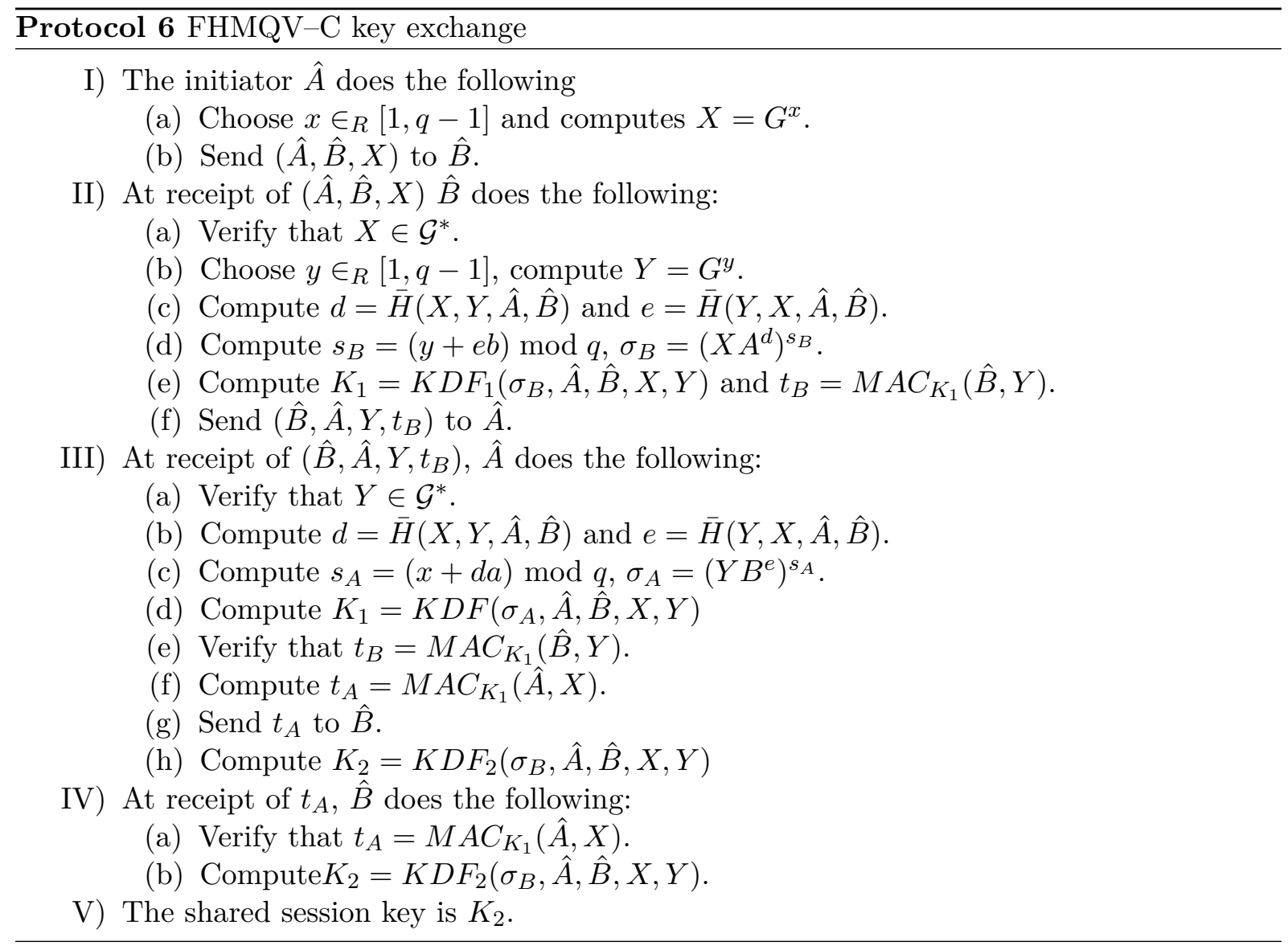

When a party $\hat{A}$ completes a FHMQV-C session with some honest peer $\hat{B}$, and with incoming ephemeral key $Y$, it is guaranteed that $Y$ was chosen and authenticated by $\hat{B}$, and that $\hat{B}$ can compute the session key it derives. The FHMQV-C protocol provides also perfect forward secrecy, the compromise of $\hat{A}$ 's static private key, does not compromise the session keys established in previous runs. This can be shown when the analysis of FHMQV is completed with the session-key expiration notion [5]. 


\section{Concluding Remarks}

We proposed a complementary analysis of the Exponential Challenge Response and Dual Exponential Challenge Response signature schemes, which are the building blocks of the HMQV protocol. On the basis of this analysis, we showed how impersonation and man in the middle attacks can be performed against the HMQV protocol, when some session specific information leakages happen.

We proposed the Full Exponential Challenge Response (FXCR) and Full Dual Exponential Challenge Response (FDCR) signature schemes, with security arguments. Using these schemes, we defined the Fully Hashed MQV (FHMQV) protocol, which preserves the efficiency and security attributes of the $(\mathrm{H}) \mathrm{MQV}$ protocols, and resists to ephemeral secret exponent leakage.

We defined a Canetti-Krawczyk type security model, based on session's tree of computations, which provides stronger reveal queries to the adversary, and showed that the FHMQV meets this security definition. The FHMQV protocol can be added with a third message, yielding the FHMQV-C protocol, which provides all the security attributes of the FHMQV protocol, added with key confirmation and perfect forward secrecy.

In a forthcoming stage, we will be interested in the analysis of relations between the security model we propose and the Canetti-Krawczyk and extended Canetti-Krawczyk security models.

Acknowledgments. The authors would like to thank Netheos R\&D for supporting this work. We also thank the EuroPKI 2009 reviewers for their useful comments.

\section{References}

[1] ANSI X9.42: Public Key Cryptography for the Financial Services Industry: Agreement of Symmetric Keys Using Discrete Logarithm Cryptography. 2001.

[2] ANSI X9.63: Public Key Cryptography for the Financial Services Industry: Key Agreement and Key Transport using Elliptic Curve Cryptography. 2001.

[3] Basin D., Cremers C.: From Dolev-Yao to Strong Adaptive Corruption: Analyzing Security in the Presence of Compromising Adversaries. Cryptology ePrint Archive, Report 2009/079, 2009 .

[4] Bellare M., Palacio A.: The Knowledge-of-Exponent Assumptions and 3-round ZeroKnowledge Protocols. Lecture Notes in Computer Science, vol. 3152, 273-289, SpringerVerlag, 2004.

[5] Canetti R., Krawczyk H.: Analysis of Key-Exchange Protocols and Their Use for Building Secure Channels. Cryptology ePrint Archive, Report 2001/040, 2001.

[6] Cremers C.: Session-state Reveal is stronger than Ephemeral Key Reveal: Attacking the NAXOS key exchange protocol. Lecture Notes in Computer Science, vol. 5536, 20-33, Springer-Verlag, 2009.

[7] Gopalakrishnan K., Thériault N., Yao C. Z.: Solving Discrete Logarithms from Partial Knowledge of the Key. Lecture Notes in Computer Science, vol. 4859, 224-237, SpringerVerlag, 2007.

[8] Hankerson D., Menezes A., Vanstone S.: Guide to Elliptic Curve Cryptography. SpringerVerlag, 2003.

[9] IEEE 1363: Standard Specifications for Public Key Cryptography. 2000.

[10] ISO/IEC IS 9798-3: Information Technology - Security techniques : Cryptography techniques based on elliptic curves - Part 3: Key Establishment. 2002. 
[11] Krawczyk H.: HMQV: A Hight Performance Secure Diffie-Hellman Protocol. Cryptology ePrint Archive, Report 2005/176, 2005.

[12] Kunz-Jacques S., Pointcheval D.: About the Security of MTI/C0 and MQV. Lecture Notes in Computer Science vol. 4116, 156-172, Springer-Verlag, 2006.

[13] LaMacchia B., Lauter K., Mityagin A.: Stronger Security of Authenticated Key Exchange. Lecture Notes in Computer Science, vol. 4784, 1-16, Springer-Verlag, 2007.

[14] Law L., Menezes A., Qu M., Solinas J., Vanstone S.: An Efficient Protocol for Authenticated Key Agreement. Designs, Codes and Cryptography, vol. 28(2), 119-134, Kluwer Academic Publishers, 2003.

[15] Leadbitter P. J., Smart N. P.: Analysis of the Insecurity of ECMQV with Partially Known Nonces. Lecture Notes in Computer Science, vol. 2851, 240-251, Springer-Verlag, 2003.

[16] Maurer U. M., Wolf S.: Diffie-Hellman Oracles. Lecture Notes in Computer Science, vol. 1109, 268-282, Springer-Verlag, 1996.

[17] Menezes A.: Another Look at HMQV. Journal of Mathematical Cryptology, vol. 1, 148-175, Walter de Gruyter, 2007.

[18] Menezes A., Ustaoglu B.: On the Importance of Public-Key Validation in the MQV and HMQV Key Agreement Protocols, Lecture Notes in Computer Science, vol. 4329, 133-147, Springer-Verlag, 2006.

[19] Menezes A., Ustaoglu B.: On Reusing Ephemeral Keys in DiffieHellman Key Agreement Protocols. Preprint, 2008 (available at http://www. cryptolounge.net/10.research.shtml).

[20] NIST Special publication 800-56: Recommendation on Key Establishment Schemes. 2003.

[21] Okamoto T., Pointcheval D.: The Gap-Problems: A new class of problems for the security of cryptographic schemes. Lecture Notes in Computer Science, vol. 1992, 104-118, SpringerVerlag, 2001.

[22] Pointcheval D.: Les preuves de connaissances et leurs preuves de sécurité. PhD thesis, Université de Caen, 1996; (in french, available at http://www.di.ens.fr/users/pointche/).

[23] Pointcheval D., Stern J.: Security Arguments for Digital Signatures and Blind Signatures. Journal of Cryptology, vol. 13, 361-396, Springer-Verlag, 2000.

[24] Pollard J. M.: Kangaroos, Monopoly and Discrete Logarithms. Journal of Cryptology, vol. 13, 437-447, Springer-Verlag, 2000.

[25] Teske E.: Square-root Algorithms for the Discrete Logarithm Problem (A survey). Public Key Cryptography and Computational Number Theory, 283-301, Walter de Gruyter, 2001.

[26] Teske E.: On Random Walks for Pollard's Rho Method. Mathematics of Computation, vol. 70, 809-825, American Mathematical Society, 2001.

[27] Ustaoglu B.: Obtaining a secure and efficient key agreement protocol from (H)MQV and NAXOS. Designs, Codes and Cryptography, vol. 46(3), 329-342, Kluwer Academic Publishers, 2008.

[28] Wang S., Cao Z., Strangio M. A., Wang L.: Cryptanalysis and Improvement of an Elliptic Curve Diffie-Hellman Key Agreement Protocol. Communications Letters, vol 12, 149-151, IEEE, 2008. 